ALEA, Lat. Am. J. Probab. Math. Stat. 16, 1105-1128 (2019)

DOI: 10.30757/ALEA.v16-41

\title{
Fractional iterated Ornstein-Uhlenbeck Processes
}

\section{Juan Kalemkerian and José Rafael León}

Universidad de la República,

Facultad de Ciencias, Montevideo, Uruguay.

E-mail address: jkalem@cmat.edu.uy

Universidad de la República,

Facultad de Ingeniería, Montevideo, Uruguay.

E-mail address: rlramos@fing.edu.uy

Abstract. We present a Gaussian process that arises from the iteration of $p$ fractional Ornstein-Uhlenbeck processes generated by the same fractional Brownian motion. When the values of the parameters defining the iteration are pairwise distinct, this iteration results in a particular linear combination of those processes. Although for $H>1 / 2$ each term of the iteration is a long memory process, we prove that when $p \geq 2$ the process obtained has short memory. We prove that the local Hölder index of the process is $H$, and obtain an explicit formula for the spectral density. We present a way to estimate the parameters and prove that the estimators are consistent and the results are asymptotically Gaussian. These processes can be used to model time series of long or short memory.

\section{Introduction}

In the field of time series, the $A R(p)$ processes are introduced as the stationary solutions of the stochastic recurrence equation

$$
X_{n}=a_{1} X_{n-1}+\ldots+a_{p} X_{n-p}+\varepsilon_{n}
$$

where the $a_{i}$ are constants and $\varepsilon_{n}$ is a Gaussian white noise. The introduction of such processes, and a fortiori of their parameters, allows some flexibility in fitting real data. However, some care must be taken to not have excessively many parameters with the consequent overfitting. To control the number of parameters, some theoretical tools must be applied, for example, Akaike's criterion. In the case of Gaussian processes in continuous time, a natural choice, generalizing the $A R(p)$ processes, is the set of processes that are the solutions of a linear stochastic differential equation of order greater than or equal to two. These processes have a rational

Received by the editors December 19th, 2018; accepted October 1st, 2019.

2010 Mathematics Subject Classification. 62M10.

Key words and phrases. Fractional Brownian motion, fractional Ornstein-Uhlenbeck process, long memory processes. 
function as spectral density. An emblematic case is the damped harmonic oscillator forced with a Gaussian white noise. As with the $A R(p)$, these processes provide some flexibility in choosing their parameters. Nevertheless, there is a drawback. Due to the fact that they are the solutions to a stochastic differential equation of order greater than or equal to two, they have at least a continuous derivative. But sometimes in practice we need models having continuous but irregular trajectories.

In the present paper we will define Gaussian processes which, like the higherorder solutions of stochastic differential equations, are defined by a set of parameters. However, we will insist that their trajectories be continuous but irregular.

These processes will be generated by a fractional noise with Hurst parameter $H$. An advantage is that in the case $H>\frac{1}{2}$, the generating process exhibits long range dependence and yet the resulting process has weak dependence. This property allows considering parameter estimation when the observation interval grows towards infinity.

In what follows we define these processes, also establishing some of their properties. We start by recalling the definition of a fractional Brownian motion.

Definition 1.1. A fractional Brownian motion with Hurst parameter $H \in(0,1]$ is an almost surely continuous centred Gaussian process $\left\{B_{H}(t)\right\}_{t \in \mathbb{R}}$ with

$$
\mathbb{E}\left(B_{H}(t) B_{H}(s)\right)=\frac{1}{2}\left(|t|^{2 H}+|s|^{2 H}-|t-s|^{2 H}\right), \quad t, s \in \mathbb{R} .
$$

When $H=1 / 2$, fractional Brownian motion becomes a standard Brownian motion. An Ornstein-Uhlenbeck process is a Gaussian process defined by $X_{t}=$ $\sigma \int_{-\infty}^{t} e^{-\lambda(t-s)} d B_{1 / 2}(t)$ for $t \in \mathbb{R}$, where $\sigma, \lambda>0$, are parameters (Uhlenbeck and Ornstein, 1930). This process is the unique stationary solution of the Langevin equation (Langevin, 1908), defined by

$$
d X_{t}=-\lambda X_{t} d t+\sigma d B_{1 / 2}(t)
$$

If we consider the Langevin equation with a fractional Brownian noise, $d X_{t}=$ $-\lambda X_{t} d t+\sigma d B_{H}(t)$, then $X_{t}=\sigma \int_{-\infty}^{t} e^{-\lambda(t-s)} d B_{H}(t)$ for $t \in \mathbb{R}$ is the unique stationary solution, see Cheridito et al. (2003). In the present paper, we use the notation $\left\{X_{t}\right\}_{t \in \mathbb{R}} \sim \operatorname{FOU}(\lambda, \sigma, H)$ for any process defined by $X_{t}=\sigma \int_{-\infty}^{t} e^{-\lambda(t-s)} d B_{H}(t)$, where $\sigma, \lambda>0, H \in(0,1]$.

If we change the process $\left\{B_{H}(t)\right\}_{t \in \mathbb{R}}$ by an unspecified process $\{y(t)\}_{t \in \mathbb{R}}$, we can define the operators $T_{\lambda}(y)(t):=\int_{-\infty}^{t} e^{-\lambda(t-s)} d y(s)$ and for each $h=0,1,2, \ldots$

$$
T_{\lambda}^{(h)}(y)(t):=\int_{-\infty}^{t} e^{-\lambda(t-s)} \frac{(-\lambda(t-s))^{h}}{h !} d y(s) .
$$

The process $\{y(t)\}_{t \in \mathbb{R}}$ are assumed to be continuous to ensure the existence of the integral defined in (1). These transformations are called the $O U$ operator with parameter $\lambda$ and the $O U$ operator of degree $h$ and parameter $\lambda$ respectively (Arratia et al., 2016).

Observe that $T_{\lambda}^{(0)}=T_{\lambda}$. Given $\left\{B_{H}(s)\right\}_{s \in \mathbb{R}}$ a fractional Brownian motion with parameter $H$, and $\lambda_{1} \neq \lambda_{2}$ real positive numbers, we define the processes $X_{t}^{(i)}:=$ $T_{\lambda_{i}}\left(\sigma B_{H}\right)(t)=\sigma \int_{-\infty}^{t} e^{-\lambda_{i}(t-s)} d B_{H}(s)$ for $i=1,2$. This is $\left\{X_{t}^{(i)}\right\}_{t \in \mathbb{R}} \sim$ $\operatorname{FOU}\left(\lambda_{i}, \sigma, H\right)$ for $i=1,2$ generated by the same fractional Brownian motion. It can be proved that the process defined by $X_{t}:=\left(T_{\lambda_{1}} \circ T_{\lambda_{2}}\right)\left(B_{H}\right)(t)$ is equal 
to $X_{t}=\frac{\lambda_{1}}{\lambda_{1}-\lambda_{2}} X_{t}^{(1)}+\frac{\lambda_{2}}{\lambda_{2}-\lambda_{1}} X_{t}^{(2)}$, which is a particular linear combination of the processes $\left\{X_{t}^{(1)}\right\}_{t \in \mathbb{R}}$ and $\left\{X_{t}^{(2)}\right\}_{t \in \mathbb{R}}$.

This implies that $\left(T_{\lambda_{1}} \circ T_{\lambda_{2}}\right)\left(B_{H}\right)=\left(T_{\lambda_{2}} \circ T_{\lambda_{1}}\right)\left(B_{H}\right)$.

In general, if we compose the operator $T_{\lambda} p$ times, we get the following equality: $T_{\lambda}^{p}=\sum_{j=0}^{p-1}\left(\begin{array}{c}p-1 \\ j\end{array}\right) T_{\lambda}^{(j)}$. If we compose the operator $T_{\lambda_{1}} p_{1}$ times, the operator $T_{\lambda_{2}}$ $p_{2}$ times,,.. , and the operator $T_{\lambda_{q}} p_{q}$ times, for pairwise distinct $\lambda_{i}$, it is possible to expand $T_{\lambda_{1}}^{p_{1}} \circ T_{\lambda_{2}}^{p_{2}} \circ \ldots \circ T_{\lambda_{q}}^{p_{q}}$ as a linear combination of the operators $T_{\lambda}^{(h)}$ (Arratia et al., 2016).

It is known that for $H>1 / 2$, every $\operatorname{FOU}(\lambda, \sigma, H)$ is a long memory process, Cheridito et al. (2003), this is $\sum_{n=-\infty}^{+\infty}|\gamma(n)|=+\infty$ where $\gamma(n)=\mathbb{E}\left(X_{0} X_{n}\right)$. We will prove, in Section 2, that if we compose at least two operators of the form $T_{\lambda}$ evaluated for a fractional Brownian motion, with Hurst parameter $H>1 / 2$, we obtain a process $\left\{X_{t}\right\}_{t \in \mathbb{R}}$ that satisfies $\sum_{n=-\infty}^{+\infty}\left|\mathbb{E}\left(X_{0} X_{n}\right)\right|<+\infty$. Further, this process has short memory.

The plan of this paper is as follows. In Section 2, we define a $\operatorname{FOU}(p)$ (this is a fractional Ornstein-Uhlenbeck process of order $p$ ), and summarize the results needed to obtain its auto-covariance function. We prove that any $\operatorname{FOU}(p)$ has almost all of its trajectories not differentiable at any point, and the parameter $H$ is the local Hölder index of the process. We also obtain its spectral density and deduce that when $p \geq 2$, it is a short memory process. In Section 3, we give explicit formulas to estimate $H$ and $\sigma$ and prove that they are consistent and the results are asymptotically Gaussian. The local behaviour of the spectral density at zero allows a consistent way to estimate the parameters of $\lambda$. In Section 4 we give a formula for the auto-covariance function of a $\operatorname{FOU}(p)$ process. Our concluding remarks are in Section 5. Section 6 contains the proofs of the results presented in Section 2.

\section{Definitions and properties}

We start with the definition of a fractional iterated Ornstein-Uhlenbeck process.

Definition 2.1. Suppose that $\left\{\sigma B_{H}(s)\right\}_{s \in \mathbb{R}}$ is a fractional Brownian motion with Hurst parameter $H$, and scale parameter $\sigma$. Suppose further that $\lambda_{1}, \lambda_{2}, \ldots, \lambda_{q}$ are distinct positive numbers and that $p_{1}, p_{2}, \ldots, p_{q} \in \mathbb{N}$ are such that $p_{1}+p_{2}+\ldots+p_{q}=p$. We define $\left\{X_{t}\right\}_{t \in \mathbb{R}}$ by

$$
X_{t}:=T_{\lambda_{1}}^{p_{1}} \circ T_{\lambda_{2}}^{p_{2}} \circ \ldots \circ T_{\lambda_{q}}^{p_{q}}\left(\sigma B_{H}\right)(t)=\sum_{i=1}^{q} K_{i}(\lambda) \sum_{j=0}^{p_{i}-1}\left(\begin{array}{c}
p_{i}-1 \\
j
\end{array}\right) T_{\lambda_{i}}^{(j)}\left(\sigma B_{H}\right)(t),
$$

where the operators $T_{\lambda_{i}}^{(j)}$ were defined in (1.1) and the numbers $K_{i}(\lambda)$ are defined by

$$
K_{i}(\lambda)=K_{i}\left(\lambda_{1}, \lambda_{2}, \ldots, \lambda_{q}\right)=\frac{1}{\prod_{j \neq i}\left(1-\lambda_{j} / \lambda_{i}\right)} .
$$

The validity of equality given by (2.1) can be found in Arratia et al. (2016).

Notation. If $\left\{X_{t}\right\}_{t \in \mathbb{R}}$ is given by equation (2.1), then we denote $\left\{X_{t}\right\}_{t \in \mathbb{R}} \sim$ FOU $\left(\lambda_{1}^{\left(p_{1}\right)}, \lambda_{2}^{\left(p_{2}\right)}, \ldots, \lambda_{q}^{\left(p_{q}\right)}, \sigma, H\right)$, or, more simply, $\left\{X_{t}\right\}_{t \in \mathbb{R}} \sim \mathrm{FOU}(p)$. 
Observe that the notation $\operatorname{FOU}\left(\lambda_{1}^{\left(p_{1}\right)}, \lambda_{2}^{\left(p_{2}\right)}, \ldots, \lambda_{q}^{\left(p_{q}\right)}, \sigma, H\right)$ implies that the parameters $\lambda_{i}$ are distinct. Also, the simplified notation $\mathrm{FOU}(p)$ means that we have taken the composition $T_{\lambda_{1}} \circ T_{\lambda_{2}} \circ \ldots \circ T_{\lambda_{p}}$ where the values of $\lambda^{\prime} s$ are arbitrary, where repetitions are allowed (this is the composition $p$ times of operators $T_{\lambda}$ ).

Remark 2.2. When $p_{1}=p_{2}=\ldots=p_{q}=1$ the process is equal to

$$
X_{t}=\left(T_{\lambda_{1}} \circ T_{\lambda_{2}} \circ \ldots \circ T_{\lambda_{q}}\right)\left(\sigma B_{H}\right)(t)=\sum_{i=1}^{q} K_{i}(\lambda) T_{\lambda_{i}}\left(\sigma B_{H}\right)(t)
$$

and we write $\left\{X_{t}\right\}_{t \in \mathbb{R}} \sim \operatorname{FOU}\left(\lambda_{1}, \lambda_{2}, \ldots, \lambda_{q}, \sigma, H\right)$.

Remark 2.3. When $q=1$, we obtain a fractional Ornstein-Uhlenbeck process $(\operatorname{FOU}(\lambda, \sigma, H))$.

Remark 2.4. Any $\operatorname{FOU}\left(\lambda_{1}^{\left(p_{1}\right)}, \lambda_{2}^{\left(p_{2}\right)}, \ldots, \lambda_{q}^{\left(p_{q}\right)}, \sigma, H\right)$, is a Gaussian, centred, and almost surely continuous process.

Any $\mathrm{FOU}(p)$ has the property that almost all its trajectories are not differentiable at any point. This fact will be used in Section 3 to obtain estimators of $H$ and $\sigma$.

Proposition 2.5. If $\left\{X_{t}\right\}_{t \in \mathbb{R}} \sim F O U(p)$, then, with probability one, its trajectories are not differentiable at any point.

In Corollary 3.3, we will see that $H$ is the local Hölder index of any $\operatorname{FOU}(p)$.

We need to define the following functions in order to obtain the main results of this paper.

$$
\begin{gathered}
f_{H}^{(1)}(x):=e^{-x}\left(\Gamma(2 H)-\int_{0}^{x} e^{s} s^{2 H-1} d s\right), \\
f_{H}^{(2)}(x):=e^{x}\left(\Gamma(2 H)-\int_{0}^{x} e^{-s} s^{2 H-1} d s\right), \\
f_{H}(x):=f_{H}^{(1)}(x)+f_{H}^{(2)}(x),
\end{gathered}
$$

where $\Gamma($.$) is the gamma function defined by \Gamma(\alpha)=\int_{0}^{+\infty} t^{\alpha-1} e^{-t} d t$.

Remark 2.6. If $H>1 / 2$, we write $f_{H}^{(1)}(x)=\frac{\Gamma(2 H)-\int_{0}^{x} e^{s} s^{2 H-1} d s}{e^{x}}$ and applying L'Hôpital rule, we obtain $f_{H}^{(1)}(x) \rightarrow-\infty$ and analogously $f_{H}^{(2)}(x) \rightarrow+\infty$ as $x \rightarrow$ $+\infty$.

As $H$ increases, the functions $f_{H}$ increase, as can be seen in Figure 2.1. Then, when $x \rightarrow+\infty$, as long as $H$ increases, the functions $f_{H}$ go to zero more slowly. The following proposition includes the properties of $f_{H}$ that will be used later on. We write $f \sim g$ as $x \rightarrow a$, when $f(x) / g(x) \rightarrow 1$ as $x \rightarrow a$.

Proposition 2.7. If $H>1 / 2, \alpha, \beta>0$, then

(1) $\alpha^{1-2 H} f_{H}^{(1)}(\alpha x)+\beta^{1-2 H} f_{H}^{(2)}(\beta x) \rightarrow 0$ when $x \rightarrow+\infty$.

(2) $\alpha^{1-2 H} f_{H}^{(1)}(\alpha x)+\beta^{1-2 H} f_{H}^{(2)}(\beta x) \sim \frac{\alpha+\beta}{\alpha \beta}(2 H-1) x^{2 H-2}$ when $x \rightarrow+\infty$.

(3) $f_{H}(x) \sim 2(2 H-1) x^{2 H-2}$ when $x \rightarrow+\infty$.

(4) $f_{H}(x)-f_{H}(0)=f_{H}(x)-2 \Gamma(2 H)=-\frac{x^{2 H}}{H}+o\left(x^{2 H}\right)$ when $x \rightarrow 0$.

(5) $f_{H}(x)=\frac{\Gamma(2 H+1) \sin (H \pi)}{2 \pi} \int_{-\infty}^{+\infty} \frac{e^{i v}|v|^{1-2 H}}{v^{2}+x^{2}} d v$. 


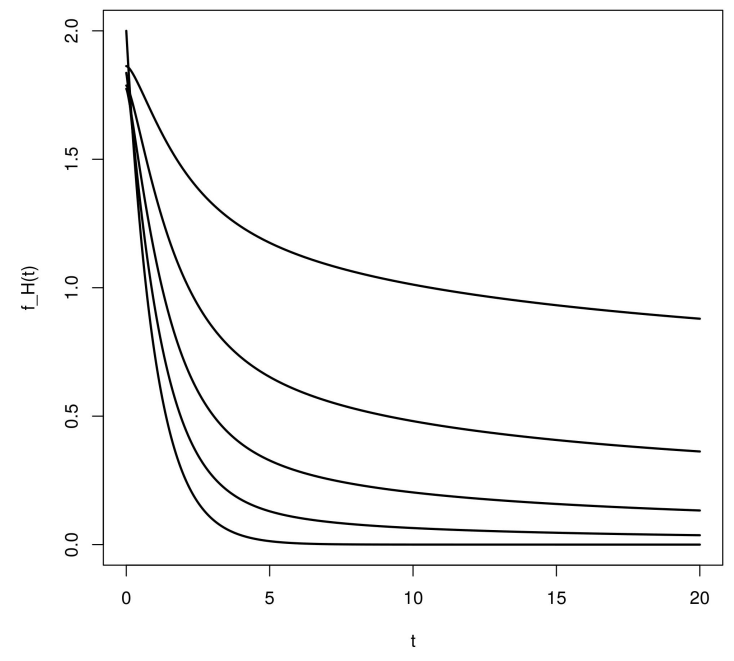

Figure 2.1. The functions $f_{H}$ for $H=0.5, \quad 0.6, \quad 0.7, \quad 0.8$ and 0.9. The lowest curve corresponds to $H=0.5$ while the highest curve corresponds to $H=0.9$.

Property 1 tells us that when $H>1 / 2, f_{H}(x) \rightarrow 0$ as $x \rightarrow+\infty$, and this fact will be used to prove property 3 . Property 3 gives the order of decay of the auto-covariance function. In particular, when $H>1 / 2$, then $\sum_{n=1}^{+\infty} f_{H}(n)=+\infty$. In Figure 2.1 it can be seen that $f_{H}$ decreases slowly to zero as $H$ increases. We will prove later, in (2.8), that the auto-covariance function of any $\operatorname{FOU}(\lambda, \sigma, H)$ can be expressed as a multiple of $f_{H}(\lambda t)$. Therefore, any $\operatorname{FOU}(\lambda, \sigma, H)$ is a long memory process for $H>1 / 2$. Property 4 will be useful for obtaining consistent estimators of $H$ and $\sigma$, independently of the parameters $\lambda$. Property 5 will be used to obtain the spectral density of any $\mathrm{FOU}(p)$.

The following proposition is the key ingredient that will allow us to express the auto-covariance function of any $\operatorname{FOU}\left(\lambda_{1}, \lambda_{2}, \ldots, \lambda_{p}, \sigma, H\right)$ as a linear combination of $f_{H}\left(\lambda_{i} t\right)$. The proof will be based on (4.1).

Proposition 2.8. Let $\left\{X_{t}^{(1)}\right\}_{t \in \mathbb{R}} \sim F O U\left(\lambda_{1}, \sigma, H\right)$ and $\left\{X_{t}^{(2)}\right\}_{t \in \mathbb{R}} \sim F O U\left(\lambda_{2}, \sigma, H\right)$ be generated by the same fractional Brownian motion $\left\{\sigma B_{H}(t)\right\}_{t \in \mathbb{R}}$. Then, for all $t$ and $H>1 / 2$,

$$
\mathbb{E}\left(X_{0}^{(1)} X_{t}^{(2)}\right)=\frac{\sigma^{2} H}{\lambda_{1}+\lambda_{2}}\left(\lambda_{1}^{1-2 H} f_{H}^{(1)}\left(\lambda_{1}|t|\right)+\lambda_{2}^{1-2 H} f_{H}^{(2)}\left(\lambda_{2}|t|\right)\right) .
$$

In particular, when $t=0$,

$\mathbb{E}\left(X_{0}^{(1)} X_{0}^{(2)}\right)=\frac{\sigma^{2} H \Gamma(2 H)}{\lambda_{1}+\lambda_{2}}\left(\lambda_{2}^{1-2 H}+\lambda_{1}^{1-2 H}\right)=\frac{\sigma^{2} \Gamma(2 H+1)}{2\left(\lambda_{1}+\lambda_{2}\right)}\left(\lambda_{1}^{1-2 H}+\lambda_{2}^{1-2 H}\right)$.

If we set $\lambda_{1}=\lambda_{2}=\lambda$ in (2.7), we obtain the auto-covariance function of any $\operatorname{FOU}(\lambda, \sigma, H)$ : 
Corollary 2.9. For any $\left\{X_{t}\right\}_{t \in \mathbb{R}} \sim F O U(\lambda, \sigma, H)$ where $H>1 / 2$,

$$
\mathbb{E}\left(X_{0} X_{t}\right)=\frac{\sigma^{2} H f_{H}(\lambda|t|)}{2 \lambda^{2 H}} .
$$

Observe that property 3 of Proposition 2.7 and (2.8), show that any $\operatorname{FOU}(\lambda, \sigma, H)$ is a long memory process.

Remark 2.10. Observe that $f_{H}(0)=2 \Gamma(2 H)$. Moreover, putting $t=0$, the well known expression for the variance of any $\operatorname{FOU}(\lambda, \sigma, H)$ appears:

$$
\mathbb{V}\left(X_{t}\right)=\frac{\sigma^{2} \Gamma(2 H+1)}{2 \lambda^{2 H}} .
$$

In Section 6 we shall prove the following proposition, which shows us that the auto-covariance function of any $F O U(p)$, where the $\lambda_{1}, \lambda_{2}, \ldots, \lambda_{p}$ are distinct, is a linear combination of the functions $f_{H}\left(\lambda_{i} t\right)$.

Proposition 2.11. If $\left\{X_{t}\right\}_{t \in \mathbb{R}} \sim F O U\left(\lambda_{1}, \lambda_{2}, \ldots, \lambda_{p}, \sigma, H\right)$ and $p \geq 2$, then

$$
\mathbb{E}\left(X_{0} X_{t}\right)=\frac{\sigma^{2} H}{2} \sum_{i=1}^{p} \frac{\lambda_{i}^{2 p-2 H-2}}{\prod_{j \neq i}\left(\lambda_{i}^{2}-\lambda_{j}^{2}\right)} f_{H}\left(\lambda_{i}|t|\right) .
$$

Remark 2.12. If $p=1$, then (2.9) is equal to (2.8).

Remark 2.13. It is possible to show that if $p \geq 2$, then $\sum_{i=1}^{p} \frac{\lambda_{i}^{2 p-4}}{\prod_{j \neq i}\left(\lambda_{i}^{2}-\lambda_{j}^{2}\right)}=0$, which allows us to see (with a little more work) that when $H>1 / 2$, then $\sum_{n=1}^{+\infty}\left|\mathbb{E}\left(X_{0} X_{n}\right)\right|$ $<+\infty$ although $\sum_{n=1}^{+\infty} f_{H}(n)=+\infty$. This fact will be also inferred from the next theorem, about the formula for the spectral density.

Observe that when $p=2$, then (2.9) says that

$$
\mathbb{E}\left(X_{0} X_{t}\right)=\frac{\sigma^{2} H}{2\left(\lambda_{1}^{2}-\lambda_{2}^{2}\right)}\left(\lambda_{1}^{2-2 H} f_{H}\left(\lambda_{1}|t|\right)-\lambda_{2}^{2-2 H} f_{H}\left(\lambda_{2}|t|\right)\right) .
$$

Using (2.9), and property 5 of the function $f_{H}$ (in Proposition 2.7) and a little more work, we obtain Theorem 2.14. The theorem gives a formula for the spectral density of the process. This result shows that if $p \geq 2$, any $\mathrm{FOU}(p)$ is a short memory process.

Theorem 2.14. If $X=\left\{X_{t}\right\}_{t \in \mathbb{R}} \sim F O U\left(\lambda_{1}^{\left(p_{1}\right)}, \lambda_{2}^{\left(p_{2}\right)}, \ldots, \lambda_{q}^{\left(p_{q}\right)}, \sigma, H\right)$ where $p_{1}+$ $p_{2}+\ldots+p_{q}=p$, then the spectral density of the process is

$$
f^{(X)}(x)=\frac{\sigma^{2} \Gamma(2 H+1) \sin (H \pi)|x|^{2 p-1-2 H}}{2 \pi \prod_{i=1}^{q}\left(\lambda_{i}^{2}+x^{2}\right)^{p_{i}}} .
$$

In particular, if $\left\{X_{t}\right\}_{t \in \mathbb{R}} \sim F O U\left(\lambda_{1}, \lambda_{2}, \ldots, \lambda_{p}, \sigma, H\right)$, then

$$
f^{(X)}(x)=\frac{\sigma^{2} \Gamma(2 H+1) \sin (H \pi)|x|^{2 p-1-2 H}}{2 \pi \prod_{i=1}^{p}\left(\lambda_{i}^{2}+x^{2}\right)} .
$$


Remark 2.15. For $H \in(1 / 2,1)$ and $p=1$, zero is a point where the spectral density of the process is singular, and we have a long memory process (because is a $\operatorname{FOU}(\lambda, \sigma, H)$ ). If $p \geq 2$, the spectral density is no longer singular at zero which suggest that the short memory property holds for such a process. This result will be formalized in the next corollary.

Remark 2.16. Observe that $f^{(X)}(0)=0$ (for $p \geq 2$ ), from which it follows that the $\mathrm{FOU}(p)$ process can be used to model anti-persistent time series. For example, our processes could be used for modeling postural control during a quiet stance by assessing the displacement of the center-of-pressure (COP) in the body. In fact, in Delignières et al. (2011), the position and the velocity of several individuals are recorded. Then, empirical evidence of the change of the correlation pattern in the velocity record of each individual is provided. In the short term, a persistent behaviour is detected and in the long term an anti-persistent behaviour is detected. One can model such a pattern by thinking of the velocity register as a fractional OU-process passing through a filter of the type $T_{\lambda_{1}}^{p_{1}} \circ T_{\lambda_{2}}^{p_{2}} \circ \ldots \circ T_{\lambda_{q}}^{p_{q}}$ in such a way that the output will be a FOU $(p)$. Nevertheless, we do not consider this matter in the present paper, it will be left to future research. Other interesting applications for anti-persistent time series can be found for instance in Ai et al. (2010) and Maxim et al. (2005).

Remark 2.17. To model non anti-persistent time series, one can develop the following idea. If $X=\left\{X_{t}\right\}_{t \in \mathbb{R}} \sim \operatorname{FOU}(p)$ and $Y=\left\{Y_{t}\right\}_{t \in \mathbb{R}}$ is a Gaussian stationary short memory process, centred, differentiable, and independent of $X$, such that $f^{(Y)}(0) \neq 0$, then $Z=X+\alpha Y$ is a centred Gaussian short memory process such that $f^{(Z)}(0)=\alpha^{2} f^{(Y)}(0)$. In this way, we have a stationary, centred Gaussian, short memory process that also has the peculiarity that $f^{(Z)}(0)$ can take any value, and the Hölder index is equal to the Hölder index of $X$. The value of $\alpha$ can be estimated from the periodogram.

Let's write (2.11) as $f^{(X)}(x)=\frac{c_{H}|x|^{2 p-1-2 H}}{\prod_{i=1}^{q}\left(\lambda_{i}^{2}+x^{2}\right)^{p_{i}}}$, where $c_{H}=\frac{\sigma^{2} \Gamma(2 H+1) \sin (H \pi)}{2 \pi}$ and consider $p \geq 2$. We have $\left|\frac{\partial f^{(X)}(x)}{\partial x^{k}}\right| \leq \frac{c t e}{x^{2}}$ for $x$ large enough.

In the other hand, observe that $\frac{\partial f^{(X)}(0)}{\partial x^{k}}=0$ for $k=1,2, \ldots, 2 p-3$, and $\frac{\partial f^{(X)}(x)}{\partial x^{2 p-2}} \sim$ $\frac{c t e}{x^{2 H-1}}$ when $x \rightarrow 0$, therefore $\int_{0}^{+\infty}\left|\frac{\partial f^{(X)}(x)}{\partial x^{2 p-2}}\right| d x<+\infty$. Then, integrating by parts $2 p-2$ times, we obtain that

$$
\rho(t)=\mathbb{E}\left(X_{0} X_{t}\right)=\int_{0}^{+\infty} \cos (t x) f^{(X)}(x) d x=\frac{-1}{t^{2 p-2}} \int_{0}^{+\infty} \cos (t x) \frac{\partial f^{(X)}(x)}{\partial x^{2 p-2}} d x .
$$

Then, we obtain the following corollary.

Corollary 2.18. If $X=\left\{X_{t}\right\}_{t \in \mathbb{R}} \sim F O U\left(\lambda_{1}^{\left(p_{1}\right)}, \lambda_{2}^{\left(p_{2}\right)}, \ldots, \lambda_{q}^{\left(p_{q}\right)}, \sigma, H\right)$ where $p_{1}+$ $p_{2}+\ldots+p_{q}=p$, for $p \geq 2$, then $|\rho(t)| \leq \frac{c}{t^{2 p-2}}$ where $c=\int_{0}^{+\infty}\left|\frac{\partial f^{(X)}(x)}{\partial x^{2 p-2}}\right| d x$ for all t. Thus $\int_{0}^{+\infty}|\rho(t)| d t<+\infty$ and the process exhibits short range dependence.

Remark 2.19. Continuing the argument that gives meaning the Corollary 2.18, observe that $\frac{\partial f^{(X)}(x)}{\partial x^{2 p-1}} \sim \frac{c t e}{x^{2 H}}$ when $x \rightarrow 0$, thus if $H>1 / 2$, we obtain $\int_{0}^{+\infty}\left|\frac{\partial f^{(X)}(x)}{\partial x^{2 p-1}}\right| d x$ $=+\infty$ and the results of Corollary 2.18 suggest that $\alpha=2 p-2$ is the maximum 
order of a bound in the form $\frac{c}{t^{\alpha}}$ for $|\rho(t)|$. Also, observe that as $p$ grows, the asymptotic decrease of the auto-covariance function of any $\mathrm{FOU}(p)$ grows.

\section{Parameter estimation}

In this section we give a consistent way to estimate the parameters of any FOU $(p)$. Firstly, we can estimate $\sigma$ and $H$ independently of the values of the $\lambda_{i}$. In addition, we will prove the consistency and asymptotic normality of the estimators. Secondly, taking advantage of the explicit formula of the spectral density, we can estimate $\lambda$ using Whittle estimators.

3.1. Estimation of $H$ and $\sigma$. If $\left\{X_{t}\right\}_{t \in \mathbb{R}}$ is a $\operatorname{FOU}(p)$ process, we define $v(t):=$ $\frac{1}{2} \mathbb{E}\left(X_{t+s}-X_{s}\right)^{2}$ the associated variogram. In Ibragimov and Rozanov (1978) it can be seen that if there is an $s \in(0,2)$ and $C>0$, such that

$$
v^{(2 D)}(t)=v^{(2 D)}(0)+C(-1)^{D}|t|^{s}+o\left(|t|^{s}\right)
$$

where $D$ is the greatest integer such that $v$ is $2 D$ times differentiable and $o\left(|t|^{s}\right)$ means that $\frac{o\left(|t|^{s}\right)}{|t|^{s}} \rightarrow 0$ when $t \rightarrow 0$, then $h=D+s / 2$ is the local Hölder index of the processes. For these processes, Istas and Lang (1997) present a procedure to estimate $C$ and $s$. In addition, they provide conditions that ensure the consistency and asymptotic normality of the estimators.

Any $\operatorname{FOU}(p)$ has almost all its trajectories everywhere non-differentiable (Proposition 2.5), therefore $D=0$. Then, the first step will be to show that $v(t)=$ $\frac{\sigma^{2}}{2}|t|^{2 H}+o\left(|t|^{2 H}\right)$, and the second step will be to look for formulas to estimate $\sigma$ and $H$ and to prove that they yield consistency and asymptotic normality.

Lemma 3.1. If $\lambda_{i} \neq \lambda_{j}$, and we define $X_{t}^{(i)}:=T_{\lambda_{i}}\left(\sigma B_{H}\right)(t)$ for each $t$, then

$$
\mathbb{E}\left(X_{t+s}^{(i)}-X_{s}^{(i)}\right)\left(X_{t+s}^{(j)}-X_{s}^{(j)}\right)=\sigma^{2}|t|^{2 H}+o\left(|t|^{2 H}\right)
$$

for all $t, s$.

Theorem 3.2. If $\left\{X_{t}\right\}_{t \in \mathbb{R}} \sim F O U(p)$, and $v(t)$ is the associated variogram, then

$$
v(t)=\frac{\sigma^{2}}{2}|t|^{2 H}+o\left(|t|^{2 H}\right) .
$$

The following corollary follows immediately from Theorem 3.2, and shows that $H$ can be interpreted as a parameter that governs the regularity of trajectories of any $\operatorname{FOU}(p)$.

Corollary 3.3. If $\left\{X_{t}\right\}_{t \in \mathbb{R}} \sim F O U(p)$ then $H$ is the local Hölder index of the process.

To obtain the estimators of $\sigma$ and $H$, we proceed as follows. First, we will call $a=\left(a_{0}, a_{1}, \ldots, a_{k}\right)$ a filter of length $k+1$ and order $L \geq 1$ if and only if the following conditions hold:

- $\sum_{i=0}^{k} a_{i} i^{l}=0$ para todo $0 \leq l \leq L-1$.

- $\sum_{i=0}^{k} a_{i} i^{L} \neq 0$. 
Observe that given $a$ a filter of order $L$ and length $k+1$, the new filter $a^{2}=$ $\left(a_{0}, 0, a_{1}, 0, a_{2}, 0, \ldots 0, a_{k}\right)$ has order $L$ and length $2 k+1$. Now, we define the quadratic variation of a sample associated to a filter $a$ as follows.

Definition 3.4. Given a filter $a$ of length $k+1$ and a sample $X_{1}, X_{2}, \ldots, X_{n}$, we define

$$
V_{n, a}:=\frac{1}{n} \sum_{i=0}^{n-k}\left(\sum_{j=0}^{k} a_{j} X_{i+j}\right)^{2} .
$$

Theorem 3.5. If $X_{\Delta}, X_{2 \Delta}, \ldots ., X_{i \Delta}, \ldots, X_{n \Delta}=X_{T}$ is an equispaced sample of the process $\left\{X_{t}\right\}_{t \in \mathbb{R}} \sim F O U(p)$ and the filter $a$ is of order $L \geq 2$ and length $k+1$, then, if $\Delta_{n}=n^{-\alpha}$ for some $\alpha$ such that $0<\alpha<\frac{1}{2(2 H-1)}$ and $T=n \Delta_{n} \rightarrow+\infty$ as $n \rightarrow+\infty$, define

$$
\begin{gathered}
\widehat{H}=\frac{1}{2} \log _{2}\left(\frac{V_{n, a^{2}}}{V_{n, a}}\right), \\
\widehat{\sigma}=\left(\frac{-2 V_{n, a}}{\Delta_{n}^{2 \widehat{H}} \sum_{i=0}^{k} \sum_{j=0}^{k} a_{i} a_{j}|i-j|^{2 \widehat{H}}}\right)^{1 / 2} .
\end{gathered}
$$

Then

$$
\begin{gathered}
(\widehat{H}, \widehat{\sigma}) \stackrel{a . s .}{\rightarrow}(H, \sigma) . \\
\sqrt{n}(\widehat{H}-H) \stackrel{w}{\rightarrow} N\left(0, \Gamma_{1}(H, \sigma, a)\right) \\
\frac{\sqrt{n}}{\log n}(\widehat{\sigma}-\sigma) \stackrel{w}{\rightarrow} N\left(0, \Gamma_{2}(H, \sigma, a)\right)
\end{gathered}
$$

3.2. Estimation of the parameters $\lambda$. Once the parameters $H$ and $\sigma$ have been estimated, taking advantage of the explicit knowledge of the spectral density, we can proceed as in Leonenko and Sakhno (2006) to estimate the rest of the parameters by using a modified Whittle contrast. Recall the spectral density of our model. If $X=\left\{X_{t}\right\}_{t \in \mathbb{R}} \sim F O U\left(\lambda_{1}^{\left(p_{1}\right)}, \ldots, \lambda_{q}^{\left(p_{q}\right)}, \sigma, H\right)$ where $\sum_{i=1}^{q} p_{i}=p$, the spectral density is

$$
f^{(X)}(x)=\frac{\sigma^{2} \Gamma(2 H+1) \sin (H \pi)|x|^{2 p-1-2 H}}{2 \pi \prod_{i=1}^{q}\left(\lambda_{i}^{2}+x^{2}\right)^{p_{i}}} .
$$

The asymptotic behaviour of this function as $|x| \rightarrow \infty$ is $O\left(|x|^{-1-2 H}\right)$. Furthermore, if $p \geq 2$, this spectral density is continuous and does not have any singularity. Indeed,

$$
\mathbb{E}\left(X_{0} X_{t}\right)=\frac{\sigma^{2} H}{2} \sum_{i=1}^{p} \frac{\lambda_{i}^{2 p-2 H-2}}{\prod_{i \neq j}\left(\lambda_{i}^{2}-\lambda_{j}^{2}\right)} f_{H}\left(\lambda_{j} t\right) .
$$

We have already shown that this covariance is integrable. Having in hand these results we can obtain in the next theorem an estimator of the parameters $\lambda$ that is consistent and asymptotically normal. 
Theorem 3.6. Suppose given $\left\{X_{t}\right\}_{t \in \mathbb{R}} \sim F O U\left(\lambda_{1}^{\left(p_{1}\right)}, \lambda_{2}^{\left(p_{2}\right)}, \ldots, \lambda_{q}^{\left(p_{q}\right)}, \sigma, H\right)$ where $\sigma$ and $H$ are known. Suppose further that $\lambda^{0}=\left(\lambda_{1}^{0}, \lambda_{2}^{0}, \ldots, \lambda_{q}^{0}\right) \in \Lambda \subset \mathbb{R}^{q}$ is the true value of the parameter, with $\lambda^{0} \in \operatorname{int}(\Lambda)$ where $\Lambda$ is compact and the process is observed in $[0, T]$ for some $T>0$. Define the following contrast process:

$$
U_{T}(\lambda)=\frac{1}{4 \pi} \int_{-\infty}^{+\infty}\left(\log f^{(X)}(x, \lambda)+\frac{I_{T}(x)}{f^{(X)}(x, \lambda)}\right) w(x) d x
$$

where $f^{(X)}(x, \lambda)$ is the spectral density of the process given in (2.11), $I_{T}(x)$ is the periodogram of the second order

$$
I_{T}(x)=\frac{1}{2 \pi T}\left|\int_{0}^{T} X_{t} e^{-i t x} d t\right|^{2}
$$

and $w(x)=\frac{|x|}{1+|x|^{b}}$ where $b>2$. Then $\widehat{\lambda}_{T}=\arg \min _{\lambda \in \Lambda} U_{T}(\lambda)$ satisfies

- $\hat{\lambda}_{T} \stackrel{P}{\rightarrow} \lambda^{0}$ when $T \rightarrow+\infty$ and

- $\sqrt{T}\left(\hat{\lambda}_{T}-\lambda^{0}\right) \stackrel{w}{\rightarrow} N_{q}\left(0, W_{1}^{-1}\left(\lambda^{0}\right) W_{2}\left(\lambda^{0}\right) W_{1}^{-1}\left(\lambda^{0}\right)\right)$ when $T \rightarrow+\infty$

where $N_{q}(.,$.$) denotes the q$-dimensional Gaussian law and the matrices $W_{1}\left(\lambda^{0}\right)$ and $W_{2}\left(\lambda^{0}\right)$ are defined by

$$
W_{1}(\lambda)=\left(w_{i j}^{(1)}(\lambda)\right)_{i, j=1, \ldots, q} \text { and } W_{2}(\lambda)=\left(w_{i j}^{(2)}(\lambda)\right)_{i, j=1, \ldots, q}
$$

where

$$
\begin{aligned}
w_{i j}^{(1)}(\lambda) & =\frac{1}{4 \pi} \int_{-\infty}^{+\infty} w(x) \frac{\partial}{\partial \lambda_{i}} \log f^{(X)}(x, \lambda) \frac{\partial}{\partial \lambda_{j}} \log f^{(X)}(x, \lambda) d x \\
w_{i j}^{(2)}(\lambda) & =\frac{1}{4 \pi} \int_{-\infty}^{+\infty} w^{2}(x) \frac{\partial}{\partial \lambda_{i}} \log f^{(X)}(x, \lambda) \frac{\partial}{\partial \lambda_{j}} \log f^{(X)}(x, \lambda) d x .
\end{aligned}
$$

Remark 3.7. It would be very interesting to prove that the following discretization

$$
U_{\Delta}=\frac{T}{2 \pi} \sum_{i=1}^{n}\left(\log f\left(x_{i}, \lambda\right)+\frac{I_{\Delta}\left(x_{i}\right)}{f\left(x_{i}, \lambda\right)}\right) w\left(x_{i}\right)\left(x_{i}-x_{i-1}\right)
$$

where $x_{i}=i \Delta, I_{\Delta}(j \Delta)=\frac{T}{2 \pi n}\left|\sum_{j=1}^{n} e^{i j \Delta} X_{j \Delta}\right|^{2}$ and $T=n \Delta \rightarrow \infty$, would give the same results as in Leonenko and Sakhno (2006). We plan to tackle this interesting problem in future work.

Remark 3.8. Let us clarify the type of convergence that we deal in the following remark. Given that the spectral density converges, then the covariances converge too. This and the fact that we consider only Gaussian processes imply the finite dimensional weak convergence. It remains to obtain that the sequence is tight. But in $C(K)$ for $K \subset \mathbb{R}$ by the Kolmogorov criterium a sequence of processes $\left\{X_{n}\right\}$ is tight if

$$
\mathbb{E}\left[\left|X_{n}(t)-X_{n}(s)\right|^{\alpha}\right] \leq C|t-s|^{\beta+1}, \text { for } \alpha, \beta>0 .
$$

But if the processes $X_{n}$ are Gaussian and stationary then we get

$$
\mathbb{E}\left[\left|X_{n}(t)-X_{n}(s)\right|^{\alpha}\right] \leq\left(2\left(1-r_{n}(t-s)\right)\right)^{\frac{\alpha}{2}} \mathbb{E}\left[N^{\alpha}\right] \leq C(2(1-r(t-s)))^{\frac{\alpha}{2}} \mathbb{E}\left[N^{\alpha}\right],
$$

where in the last inequality we have used the convergence of the covariance, then the continuity of the limit process give the tightness by taking $\alpha>2$. 
Remark 3.9. If we take $\lambda_{1}=\lambda$ and make $\lambda_{2} \rightarrow 0$ in (2.10) we obtain (2.8). This implies that $\operatorname{FOU}\left(\lambda_{1}, \lambda_{2}, \sigma, H\right) \stackrel{\mathcal{L}}{\rightarrow} \operatorname{FOU}(\lambda, \sigma, H)$ where $\mathcal{L}$ denotes the weak convergence of processes of the remark 3.8. We can sketch an ideal semiparametric test of hypothesis: long range dependence versus short range dependence. The $H_{0}$ hypothesis is that our $\operatorname{FOU}\left(\lambda_{1}, \lambda_{2}, \sigma, H\right)$ process has $\lambda_{2}=0$, this is it has long range dependence. By using the spectral estimator that has already studied in the present work a rejection region can be defined. Moreover, the asymptotic power of the test can be obtained under the sequence of contiguous alternatives $\lambda_{2, n} \rightarrow 0$.

\section{Computing the auto-covariance function}

Now, we compute the auto-covariance function of any FOU $(p)$ process. For this we need the following formula, whose proof can be found in Pipiras and Taqqu (2000): if $H \in(1 / 2,1)$ and

$$
f, g \in\left\{f: \mathbb{R} \rightarrow \mathbb{R}: \iint_{\mathbb{R}^{2}}|f(u) f(v)||u-v|^{2 H-2} d u d v<+\infty\right\},
$$

then

$$
\begin{aligned}
& \mathbb{E}\left(\int_{-\infty}^{+\infty} f(u) d B_{H}(u) \int_{-\infty}^{+\infty} g(v) d B_{H}(v)\right) \\
& =H(2 H-1) \int_{-\infty}^{+\infty} f(u) d u \int_{-\infty}^{+\infty} g(v)|u-v|^{2 H-2} d v
\end{aligned}
$$

We start with

$$
\begin{aligned}
& \gamma(t)=\mathbb{E}\left(X_{t} X_{0}\right) \\
& =\mathbb{E} \sum_{h=1}^{q} K_{h}(\lambda) \sum_{j=0}^{p_{h}-1}\left(\begin{array}{c}
p_{h}-1 \\
j
\end{array}\right) T_{\lambda_{h}}^{(j)}\left(\sigma B_{H}\right)(t) \sum_{h^{\prime}=1}^{q} K_{h^{\prime}}(\lambda) \sum_{j^{\prime}=0}^{p_{h^{\prime}}-1}\left(\begin{array}{c}
p_{h^{\prime}}-1 \\
j^{\prime}
\end{array}\right) T_{\lambda_{h^{\prime}}}^{\left(j^{\prime}\right)}\left(\sigma B_{H}\right)(0) \\
& =\sum_{i=1}^{q} \sum_{i^{\prime}=1}^{q} \sum_{j=0}^{p_{i}-1} \sum_{j^{\prime}=0}^{p_{i^{\prime}}-1} K_{i}(\lambda)\left(\begin{array}{c}
p_{i}-1 \\
j
\end{array}\right) K_{i^{\prime}}(\lambda)\left(\begin{array}{c}
p_{i^{\prime}}-1 \\
j^{\prime}
\end{array}\right) \mathbb{E} T_{\lambda_{i}}^{(j)}\left(\sigma B_{H}\right)(t) T_{\lambda_{i^{\prime}}}^{\left(j^{\prime}\right)}\left(\sigma B_{H}\right)(0) . \\
& \text { Define } \gamma_{\lambda, \lambda^{\prime}}^{\left(j, j^{\prime}\right)}(t):=\mathbb{E} T_{\lambda}^{(j)}\left(\sigma B_{H}\right)(t) T_{\lambda^{\prime}}^{\left(j^{\prime}\right)}\left(\sigma B_{H}\right)(0), \text { then } \\
& \gamma(t)=\sum_{i, i^{\prime}=1}^{q} K_{i}(\lambda) K_{i^{\prime}}(\lambda) \sum_{j=0}^{p_{i}-1} \sum_{j^{\prime}=0}^{p_{i^{\prime}}-1}\left(\begin{array}{c}
p_{i}-1 \\
j
\end{array}\right)\left(\begin{array}{c}
p_{i^{\prime}}-1 \\
j^{\prime}
\end{array}\right) \gamma_{\lambda_{i}, \lambda_{i^{\prime}}}^{\left(j, j^{\prime}\right)}(t) .
\end{aligned}
$$

Now, we compute $\gamma_{\lambda, \lambda^{\prime}}^{\left(j, j^{\prime}\right)}(t)$.

$$
\begin{gathered}
\gamma_{\lambda, \lambda^{\prime}}^{\left(j, j^{\prime}\right)}(t)=\mathbb{E} T_{\lambda}^{(j)}\left(\sigma B_{H}\right)(t) T_{\lambda^{\prime}}^{\left(j^{\prime}\right)}\left(\sigma B_{H}\right)(0) \\
=\sigma^{2} \mathbb{E} \int_{-\infty}^{t} e^{-\lambda(t-u)} \frac{(-\lambda(t-u))^{j}}{j !} d B_{H}(u) \int_{-\infty}^{0} e^{\lambda^{\prime} v} \frac{\lambda^{j^{\prime} j^{\prime}} v^{j^{\prime}}}{j^{\prime} !} d B_{H}(v) .
\end{gathered}
$$

Using (4.1), we obtain that (4.3) is equal to

$$
\sigma^{2} H(2 H-1) \int_{-\infty}^{t} e^{-\lambda(t-u)} \frac{(-\lambda(t-u))^{j}}{j !} d u \int_{-\infty}^{0} e^{\lambda^{\prime} v} \frac{\lambda^{\prime j^{\prime}} v^{j^{\prime}}}{j^{\prime} !}|u-v|^{2 H-2} d v
$$




$$
\begin{gathered}
=\frac{\sigma^{2} H(2 H-1) \lambda^{j} \lambda^{j^{\prime}}}{j ! j^{\prime} !} \int_{-\infty}^{t} e^{-\lambda(t-u)}(u-t)^{j} d u \int_{-\infty}^{0} e^{\lambda^{\prime} v} v^{j^{\prime}}|u-v|^{2 H-2} d v \\
\stackrel{w=u-t}{=} \frac{\sigma^{2} H(2 H-1) \lambda^{j} \lambda^{\prime j^{\prime}}}{j ! j^{\prime} !} \int_{-\infty}^{0} e^{\lambda w} w^{j} d w \int_{-\infty}^{0} e^{\lambda^{\prime} v} v^{j^{\prime}}|w+t-v|^{2 H-2} d v \\
=\frac{\sigma^{2} H(2 H-1) \lambda^{j} \lambda^{\prime j^{\prime}}(-1)^{j+j^{\prime}}}{j ! j^{\prime} !} \int_{0}^{+\infty} e^{-\lambda w} w^{j} d w \int_{0}^{+\infty} e^{-\lambda^{\prime} v} v^{j^{\prime}}|v+t-w|^{2 H-2} d v .
\end{gathered}
$$

Remark 4.1. In the very interesting paper of Kaarakka and Salminen (2011) it is introduced a certain stationary mean zero Gaussian process $\hat{Y}^{(1)}(t)$, defined for $t \in \mathbb{R}$, that exhibes long range dependence and certain autosimilarity property. They use $\hat{Y}^{(1)}(t)$ as a bilateral noise. Then the following SDE is studied

$$
d U^{(D, \gamma)}(t)=-\gamma U^{(D, \gamma)}(t) d t+d \hat{Y}^{(1)}(t)
$$

proving that a Gaussian stationary solution exists. This process is called a fractional Ornstein-Uhlenbeck (OU) of the second kind. The authors show afterwards that its covariance behaves

$$
\mathbb{E}\left(U^{(D, \gamma)}(t) U^{(D, \gamma)}(0)\right)=O\left(e^{-\min \left(\gamma, \frac{(1-H)}{H}\right) t}\right) \text { as } t \rightarrow \infty .
$$

Hence, a short range dependence of exponential type holds. A comparison can be made with our $F O U(p)$ processes. First at all our processes exhibit a short range dependence also but of polynomial type. Another interesting difference is that the $F O U(p)$ processes allow fitting several parameters, instead of two as is the case of fractional OU of the second kind. We can pushforward our commentaries by considering other stationary mean zero Gaussian $X$ processes, those whose covariance function is given by $r_{X}(t)=e^{-\gamma|t|^{H}}$ for $0<H<2$. These processes although do not have a SDE representation, are short range dependent of exponential type too. Besides, the three processes cited here have the same type of local smoothness.

\section{Conclusions}

In this paper we have presented a family of Gaussian processes that arise from the iteration of $p$ fractional Ornstein-Uhlenbeck processes generated by the same fractional Brownian motion. When the $\lambda_{i}$ are distinct, this iteration results in a particular linear combination of fractional Ornstein-Uhlenbeck processes. We proved that when $H>1 / 2$ and the $\lambda_{i}$ are distinct, the auto-covariance function of the process can be expressed as a linear combination of the auto-covariance functions of each $\operatorname{FOU}\left(\lambda_{i}, \sigma, H\right)$. We have obtained an explicit formula for the spectral density of the process, which allows us to deduce that although every fractional Ornstein-Uhlenbeck process with $H>1 / 2$ is a long memory process, for $p \geq 2$, the iteration results in a short memory process. As $p$ grows the decreasing of the autocovariance function of any $\mathrm{FOU}(p)$ grows. We have proposed consistent estimators for all the parameters. Modelling short memory continuous time series using the FOU $(p)$ processes will yield some advantages, because of the possibility to choose several of the parameters $\lambda$ and moreover by using $H$ to measure the regularity of the trajectories. Lastly, the $\mathrm{FOU}(p)$ process (when choosing an appropriate value of $p$ ) can be used to model time series of long or short memory. 


\section{Proofs}

Proof of Proposition 2.5: We start showing that $\sum_{j=1}^{q} K_{j}(\lambda)=1$.

Define $p(x)=\sum_{j=1}^{q} K_{j}(\lambda) \prod_{i \neq j}\left(1+\lambda_{i} x\right)$. Observe that $p$ is a polynomial of degree at most $q-1$ and $p(0)=\sum_{j=1}^{q} K_{j}(\lambda)$. Also observe that $p(x)=$ $\sum_{j=1}^{q} \prod_{i \neq j} \frac{1+\lambda_{i} x}{1-\lambda_{i} / \lambda_{j}}$ and $p\left(\frac{-1}{\lambda_{h}}\right)=1$ for all $h=1,2, \ldots, q$. Then $p$ necessary is constant and it follows that $\sum_{j=1}^{q} K_{j}(\lambda)=1$.

For any $j=1,2,3, .$. , we integrate by parts and obtain that

$$
T_{\lambda}^{(j)}\left(B_{H}\right)(t)=\frac{(-\lambda)^{j+1}}{j !} \int_{-\infty}^{t} B_{H}(s) e^{-\lambda(t-s)}(t-s)^{j-1}(t-s-j) d s .
$$

If $j=0, T_{\lambda}\left(B_{H}\right)(t)=B_{H}(t)-\lambda \int_{-\infty}^{t} B_{H}(s) e^{-\lambda(t-s)} d s$. Then $T_{\lambda}^{(j)}\left(B_{H}\right)$ is differentiable for $j=1,2,3, \ldots$ and is not differentiable for $j=0$ at any point.

$$
\begin{aligned}
& \sum_{i=1}^{q} K_{i}(\lambda) \sum_{j=0}^{p_{i}-1}\left(\begin{array}{c}
j \\
p_{i}-1
\end{array}\right) T_{\lambda_{i}}^{(j)}\left(B_{H}\right)(t) \\
= & \sum_{i=1}^{q} K_{i}(\lambda)\left(T_{\lambda_{i}}\left(B_{H}\right)(t)+\sum_{j=1}^{p_{i}-1}\left(\begin{array}{c}
j \\
p_{i}-1
\end{array}\right) T_{\lambda_{i}}^{(j)}\left(B_{H}\right)(t)\right) \\
= & \sum_{i=1}^{q} K_{i}(\lambda) T_{\lambda_{i}}\left(B_{H}\right)(t)+\sum_{i=1}^{q} K_{i}(\lambda) \sum_{j=1}^{p_{h}-1}\left(\begin{array}{c}
j \\
p_{i}-1
\end{array}\right) T_{\lambda_{i}}^{(j)}\left(B_{H}\right)(t) .
\end{aligned}
$$

Using that $\sum_{h=1}^{q} K_{h}(\lambda)=1$, we deduce that

$$
\begin{gathered}
\sum_{i=1}^{q} K_{i}(\lambda) T_{\lambda_{i}}\left(B_{H}\right)(t)=\sum_{i=1}^{q} K_{i}(\lambda)\left(B_{H}(t)-\lambda_{i} \int_{-\infty}^{t} B_{H}(s) e^{-\lambda_{i}(t-s)} d s\right) \\
=B_{H}(t)-\sum_{i=1}^{q} K_{i}(\lambda) \lambda_{i} \int_{-\infty}^{t} B_{H}(s) e^{-\lambda_{i}(t-s)} d s
\end{gathered}
$$

and so, with probability one, the trajectories of $X$ are not differentiable at any point. This concludes the proof.

Proof of Proposition 2.7: $\quad$ (1) It is enough to prove that

$$
\frac{-\alpha^{1-2 H} \int_{0}^{\alpha x} e^{s} s^{2 H-1} d s+\beta^{1-2 H} e^{(\alpha+\beta) x} \int_{\beta x}^{+\infty} e^{-s} s^{2 H-1} d s}{e^{\alpha x}} \rightarrow 0 .
$$

We apply L'Hôpital's rule twice to obtain

$$
\begin{gathered}
\lim _{x \rightarrow+\infty} \frac{\alpha+\beta}{\alpha} \frac{-e^{-\beta x} x^{2 H-1}+\beta^{1-2 H} \int_{\beta x}^{+\infty} e^{-s} s^{2 H-1} d s}{e^{-\beta x}}= \\
\lim _{x \rightarrow+\infty} \frac{\alpha+\beta}{\alpha \beta}(2 H-1) x^{2 H-2} \rightarrow 0 .
\end{gathered}
$$


(2) Since $\alpha^{1-2 H} f_{H}^{(1)}(\alpha x)+\beta^{1-2 H} f_{H}^{(2)}(\beta x) \rightarrow 0$ and $e^{-x} / x^{2-2 H} \rightarrow 0$ as $x \rightarrow$ $+\infty$, we can apply L'Hôpital's rule and get

$$
\begin{aligned}
& \lim _{x \rightarrow+\infty} \frac{\alpha^{1-2 H} f_{H}^{(1)}(\alpha x)+\beta^{1-2 H} f_{H}^{(2)}(\beta x)}{x^{2 H-2}} \\
& =\lim _{x \rightarrow+\infty} \frac{\beta^{1-2 H} e^{(\alpha+\beta) x} \int_{\beta x}^{+\infty} e^{-s} s^{2 H-1} d s-\alpha^{1-2 H} \int_{0}^{\alpha x} e^{s} s^{2 H-1} d s}{e^{\alpha x} x^{2 H-2}} \\
& =\lim _{x \rightarrow+\infty} \frac{\alpha+\beta}{\alpha} \frac{-x^{2 H-1}+\beta^{1-2 H} e^{\beta x} \int_{\beta x}^{+\infty} e^{-s} s^{2 H-1} d s}{x^{2 H-2}}=\frac{\alpha+\beta}{\alpha \beta}(2 H-1)
\end{aligned}
$$

where in the last equality was from applying L'Hôpital's rule again.

(3) In property 2 , put $\alpha=\beta=\lambda$. Then we get that

$$
f_{H}(\lambda x)=f_{H}^{(1)}(\lambda x)+f_{H}^{(2)}(\lambda x) \sim 2(2 H-1)(\lambda x)^{2 H-2}
$$

where $x \rightarrow+\infty$.

(4) Furthermore,

$$
\begin{aligned}
& f_{H}(x)-f_{H}(0)=f_{H}(x)-2 \Gamma(2 H) \\
& =\Gamma(2 H)\left(e^{x}+e^{-x}-2\right)-e^{-x} \int_{0}^{x} e^{s} s^{2 H-1} d s-e^{x} \int_{0}^{x} e^{-s} s^{2 H-1} d s \\
& =o\left(x^{2 H}\right)-e^{x} \sum_{n=0}^{+\infty} \frac{x^{n+2 H}}{n !(n+2 H)}-e^{-x} \sum_{n=0}^{+\infty} \frac{(-1)^{n} x^{n+2 H}}{n !(n+2 H)}=o\left(x^{2 H}\right)-\frac{x^{2 H}}{H} .
\end{aligned}
$$

(5) Cheridito et al. (2003) have shown that if $X_{t} \sim \operatorname{FOU}(\lambda, \sigma, H)$, then

$$
\rho(t)=\mathbb{E}\left(X_{0} X_{t}\right)=\frac{\sigma^{2} \Gamma(2 H+1) \sin (H \pi)}{2 \pi} \int_{-\infty}^{+\infty} \frac{e^{i t x}|x|^{1-2 H}}{\lambda^{2}+x^{2}} d x .
$$

But, due to (2.8), $\rho(t)=\frac{\sigma^{2} H f_{H}(\lambda t)}{2 \lambda^{2 H}}$, and then we deduce that

$$
f_{H}(\lambda t)=\frac{2 \Gamma(2 H) \sin (H \pi) \lambda^{2 H}}{H \pi} \int_{-\infty}^{+\infty} \frac{e^{i t x}|x|^{1-2 H}}{\lambda^{2}+x^{2}} d x .
$$

Finally, if we make the change of variable $x=\lambda v$, we obtain the result. This concludes the proof.

Observe that in the proof of property 5, we use (2.8) that is a corollary of Proposition 2.8, but the proof of Proposition 2.8 is independent of any property of $f_{H}$.

Proof of Proposition 2.8:

$$
\mathbb{E}\left(X_{t}^{(1)} X_{s}^{(2)}\right)=\sigma^{2} E\left(\int_{-\infty}^{t} e^{-\lambda_{1}(t-u)} d B_{H}(u) \int_{-\infty}^{s} e^{-\lambda_{2}(s-v)} d B_{H}(v)\right) .
$$

As $H>1 / 2$, we can apply (4.1), and so

$$
\mathbb{E}\left(X_{t}^{(1)} X_{s}^{(2)}\right)=\sigma^{2} H(2 H-1) \int_{-\infty}^{t} e^{-\lambda_{1}(t-u)} d u \int_{-\infty}^{s} e^{-\lambda_{2}(s-v)}|u-v|^{2 H-2} d v
$$


Now we make the change of variable $w=t-u, z=s-v$ and get that

$$
\mathbb{E}\left(X_{t}^{(1)} X_{s}^{(2)}\right)=\sigma^{2} H(2 H-1) \int_{0}^{+\infty} e^{-\lambda_{1} w} d w \int_{0}^{+\infty} e^{-\lambda_{2} z}|t-w+z-s|^{2 H-2} d z .
$$

Then, $\mathbb{E}\left(X_{t}^{(1)} X_{s}^{(2)}\right)$ only depends on $t-s$, so we only need to find a formula for $\mathbb{E}\left(X_{0}^{(1)} X_{t}^{(2)}\right)$.

$$
\mathbb{E}\left(X_{0}^{(1)} X_{t}^{(2)}\right)=\sigma^{2} H(2 H-1) \int_{0}^{+\infty} d w \int_{0}^{+\infty} e^{-\lambda_{1} w-\lambda_{2} z}|z-w-t|^{2 H-2} d z
$$

then, after doing the change of variable $h=\lambda_{1} w+\lambda_{2} z$ in the integral in $z$, this is equal to

$$
\begin{gathered}
\frac{\sigma^{2} H(2 H-1)}{\lambda_{2}} \int_{0}^{+\infty} d w \int_{\lambda_{1} w}^{+\infty} e^{-h}\left|\frac{h-\lambda_{1} w}{\lambda_{2}}-w-t\right|^{2 H-2} d h= \\
\frac{\sigma^{2} H(2 H-1)}{\lambda_{2}^{2 H-1}} \int_{0}^{+\infty} d w \int_{\lambda_{1} w}^{+\infty} e^{-h}\left|h-\left(\lambda_{1}+\lambda_{2}\right) w-\lambda_{2} t\right|^{2 H-2} d h= \\
\frac{\sigma^{2} H(2 H-1)}{\lambda_{2}^{2 H-1}} \int_{0}^{+\infty} e^{-h} d h \int_{0}^{h / \lambda_{1}}\left|h-\left(\lambda_{1}+\lambda_{2}\right) w-\lambda_{2} t\right|^{2 H-2} d w .
\end{gathered}
$$

Now, we continue the calculation in the case $t \geq 0$, distinguishing three regions according to the absolute value that apears in the last integral. Then, we get that (6.1) is equal to

$$
\begin{aligned}
& \frac{\sigma^{2} H(2 H-1)}{\lambda_{2}^{2 H-1}} \int_{0}^{\lambda_{2} t} e^{-h} d h \int_{0}^{h / \lambda_{1}}\left(\left(\lambda_{1}+\lambda_{2}\right) w+\lambda_{2} t-h\right)^{2 H-2} d w+ \\
& \frac{\sigma^{2} H(2 H-1)}{\lambda_{2}^{2 H-1}} \int_{\lambda_{2} t}^{+\infty} e^{-h} d h \int_{0}^{\frac{h-\lambda_{2} t}{\lambda_{1}+\lambda_{2}}}\left(h-\left(\lambda_{1}+\lambda_{2}\right) w-\lambda_{2} t\right)^{2 H-2} d w+ \\
& \frac{\sigma^{2} H(2 H-1)}{\lambda_{2}^{2 H-1}} \int_{\lambda_{2} t}^{+\infty} e^{-h} d h \int_{\frac{h-\lambda_{2} t}{\lambda_{1}+\lambda_{2}}}^{h / \lambda_{1}}\left(\left(\lambda_{1}+\lambda_{2}\right) w+\lambda_{2} t-h\right)^{2 H-2} d w .
\end{aligned}
$$

Now we make $s=\lambda_{2} t-h$ in the first summand and $s=h+\lambda_{1} t$ in second, and we get

$$
\begin{gathered}
\frac{\sigma^{2} H}{\lambda_{1}+\lambda_{2}}\left(e^{-\lambda_{2} t} \Gamma(2 H) \lambda_{2}^{1-2 H}-\lambda_{2}^{1-2 H} \int_{0}^{\lambda_{2} t} e^{-h}\left(\lambda_{2} t-h\right)^{2 H-1} d h\right)+ \\
\frac{\sigma^{2} H}{\lambda_{1}+\lambda_{2}} \lambda_{1}^{1-2 H} \int_{0}^{+\infty} e^{-h}\left(h+\lambda_{1} t\right)^{2 H-1} d h \\
=\frac{\sigma^{2} H}{\lambda_{1}+\lambda_{2}}\left(e^{-\lambda_{2} t} \Gamma(2 H) \lambda_{2}^{1-2 H}-\lambda_{2}^{1-2 H} e^{-\lambda_{2} t} \int_{0}^{\lambda_{2} t} e^{s} s^{2 H-1} d s\right)+ \\
\frac{\sigma^{2} H}{\lambda_{1}+\lambda_{2}} \lambda_{1}^{1-2 H} e^{\lambda_{1} t} \int_{\lambda_{1} t}^{+\infty} e^{-s} s^{2 H-1} d s \\
=\frac{\sigma^{2} H}{\lambda_{1}+\lambda_{2}}\left(\lambda_{2}^{1-2 H} f_{H}^{(2)}\left(\lambda_{2} t\right)+\lambda_{1}^{1-2 H}\left[e^{\lambda_{1} t} \Gamma(2 H)-e^{\lambda_{1} t} \int_{0}^{\lambda_{1} t} e^{-s} s^{2 H-1} d s\right]\right) \\
=\frac{\sigma^{2} H}{\lambda_{1}+\lambda_{2}}\left(\lambda_{2}^{1-2 H} f_{H}^{(2)}\left(\lambda_{2} t\right)+\lambda_{1}^{1-2 H} f_{H}^{(1)}\left(\lambda_{1} t\right)\right) .
\end{gathered}
$$


The case $t \leq 0$, is treated similarly. This concludes the proof.

To prove Proposition 2.11, we need the following lemma.

Lemma 6.1. If $\lambda_{1}, \lambda_{2}, \ldots, \lambda_{p}$ are distinct positive reals numbers, then

$$
K_{i}+2 \lambda_{i} \sum_{j \neq i} \frac{K_{j}}{\lambda_{i}+\lambda_{j}}=\frac{\lambda_{i}^{p-1}}{\prod_{j \neq i}\left(\lambda_{i}+\lambda_{j}\right)} \text { for } i=1,2,3, \ldots, p
$$

where $K_{i}=K_{i}(\lambda)$ for $i=1,2, \ldots, p$ are defined in (2.2).

Proof:

To obtain the result, it is enough to show that

$$
K_{1}+2 \lambda_{1} \sum_{j=2}^{p} \frac{K_{j}}{\lambda_{i}+\lambda_{j}}=\frac{\lambda_{1}^{p-1}}{\prod_{j=2}^{p}\left(\lambda_{1}+\lambda_{j}\right)} .
$$

Because, $K_{i}=\frac{\lambda_{i}^{p-1}}{\prod_{j \neq i}^{p}\left(\lambda_{i}-\lambda_{j}\right)}$, then (6.2) is equal to

$$
\frac{\lambda_{1}^{p-1}}{\prod_{j=2}^{p}\left(\lambda_{1}-\lambda_{j}\right)}+2 \lambda_{1} \sum_{j=2}^{p} \frac{K_{j}}{\lambda_{1}+\lambda_{j}}=\frac{\lambda_{1}^{p-1}}{\prod_{j=2}^{p}\left(\lambda_{1}+\lambda_{j}\right)}
$$

which is equivalent to proving that (if we write $x=\lambda_{1}$ )

$$
\frac{x^{p-2}}{\prod_{j=2}^{p}\left(x-\lambda_{j}\right)}-\frac{x^{p-2}}{\prod_{j=2}^{p}\left(x+\lambda_{j}\right)}=-2 \sum_{j=2}^{p} \frac{K_{j}}{x+\lambda_{j}} .
$$

In fact, we can develop the quotient in simple fractions, and obtain that (6.3) is equal to

$$
\begin{gathered}
\frac{x^{p-2}}{\prod_{j=2}^{p}\left(x-\lambda_{j}\right)}-\frac{x^{p-2}}{\prod_{j=2}^{p}\left(x+\lambda_{j}\right)}= \\
=\sum_{i=2}^{p}\left(\frac{\lambda_{i}^{p-2}}{\prod_{j \neq i}\left(\lambda_{i}-\lambda_{j}\right)} \frac{1}{x-\lambda_{i}}-\frac{\left(-\lambda_{i}\right)^{p-2}}{\prod_{j \neq i}\left(\lambda_{j}-\lambda_{i}\right)} \frac{1}{x+\lambda_{i}}\right) \\
=\sum_{i=2}^{p}\left(\frac{\lambda_{i}^{p-2} 2 \lambda_{i}}{\prod_{j \neq i}\left(\lambda_{i}-\lambda_{j}\right)} \frac{1}{\left(x-\lambda_{i}\right)\left(x+\lambda_{i}\right)}\right) \\
=-2 \sum_{i=2}^{p} \frac{\lambda_{i}^{p-1}}{\left(\lambda_{i}-x\right) \prod_{j \neq i}\left(\lambda_{i}-\lambda_{j}\right)} \frac{1}{\left(x+\lambda_{i}\right)}=-2 \sum_{j=2}^{p} \frac{K_{j}}{x+\lambda_{j}} .
\end{gathered}
$$

This concludes the proof. 
Proof of Proposition 2.11: We start with (4.2) in the case $p_{1}=p_{2}=\ldots \ldots=p_{q}=1$,

$$
\gamma(t)=\mathbb{E}\left(X_{t} X_{0}\right)=\sum_{i, j=1}^{q} K_{i} K_{j} \gamma_{\lambda_{i}, \lambda_{j}}^{(0,0)}(t)
$$

and using (2.7)

$$
\begin{gathered}
\gamma(t)=\sigma^{2} H \sum_{i, j=1}^{q} K_{i} K_{j} \frac{\left(\lambda_{i}^{1-2 H} f_{H}^{(1)}\left(\lambda_{i} t\right)+\lambda_{j}^{1-2 H} f_{H}^{(2)}\left(\lambda_{j} t\right)\right)}{\lambda_{i}+\lambda_{j}} \\
=\sigma^{2} H\left(\sum_{i, j=1}^{p} \frac{K_{i}^{2}}{2 \lambda_{i}} \lambda_{i}^{1-2 H} f_{H}\left(\lambda_{i} t\right)+\sum_{i=1}^{p} K_{i} \lambda_{i}^{1-2 H} f_{H}^{(1)}\left(\lambda_{i} t\right) \sum_{j \neq i} \frac{K_{j}}{\lambda_{i}+\lambda_{j}}\right)+ \\
\sigma^{2} H \sum_{i=1}^{p} K_{i} \lambda_{i}^{1-2 H} f_{H}^{(2)}\left(\lambda_{i} t\right) \sum_{j \neq i} \frac{K_{j}}{\lambda_{i}+\lambda_{j}} \\
=\sigma^{2} H \sum_{i=1}^{p} K_{i} \lambda_{i}^{-2 H} f_{H}\left(\lambda_{i} t\right)\left(\frac{K_{i}}{2}+\lambda_{i} \sum_{j \neq i} \frac{K_{j}}{\lambda_{i}+\lambda_{j}}\right)
\end{gathered}
$$

Now, using Lemma 6.1, the last expression is equal to

$$
\begin{aligned}
& \frac{\sigma^{2} H}{2} \sum_{i=1}^{p} K_{i} \lambda_{i}^{-2 H} f_{H}\left(\lambda_{i} t\right) \frac{\lambda_{i}^{p-1}}{\prod_{j \neq i}\left(\lambda_{i}+\lambda_{j}\right)} \\
& =\frac{\sigma^{2} H}{2} \sum_{i=1}^{p} \frac{\lambda_{i}^{p-1}}{\prod_{j \neq i}\left(\lambda_{i}-\lambda_{j}\right)} \lambda_{i}^{-2 H} f_{H}\left(\lambda_{i} t\right) \frac{\lambda_{i}^{p-1}}{\prod_{j \neq i}\left(\lambda_{i}+\lambda_{j}\right)} \\
& =\frac{\sigma^{2} H}{2} \sum_{i=1}^{p} \frac{\lambda_{i}^{2 p-2 H-2}}{\prod_{j \neq i}\left(\lambda_{i}^{2}-\lambda_{j}^{2}\right)} f_{H}\left(\lambda_{i} t\right) .
\end{aligned}
$$

This concludes the proof.

The expression for the spectral density is a consequence of the equalities established in the following two lemmas.

Lemma 6.2. If $\lambda_{1}, \lambda_{2}, \ldots, \lambda_{p}, p \geq 2$, are distinct positive real numbers, and $x \neq \lambda_{i}$ for all $i=1,2, \ldots, p$ then

$$
\frac{x^{2 p-2}}{\prod_{i=1}^{p}\left(x^{2}-\lambda_{j}^{2}\right)}=\sum_{i=1}^{p} \frac{\lambda_{i}^{2 p-2}}{\prod_{j \neq i}\left(\lambda_{i}^{2}-\lambda_{j}^{2}\right)} \frac{1}{x^{2}-\lambda_{i}^{2}} .
$$

Proof: A decomposition into simple fractions yields

$$
\begin{gathered}
\frac{x^{2 p-2}}{\prod_{i=1}^{p}\left(x^{2}-\lambda_{j}^{2}\right)}=\frac{x^{2 p-2}}{\prod_{i=1}^{p}\left(x-\lambda_{j}\right)\left(x+\lambda_{j}\right)}= \\
\sum_{i=1}^{p}\left(\frac{\lambda_{i}^{2 p-2}}{2 \lambda_{i} \prod_{j \neq i}\left(\lambda_{i}^{2}-\lambda_{j}^{2}\right)} \frac{1}{x-\lambda_{i}}-\frac{\lambda_{i}^{2 p-2}}{2 \lambda_{i} \prod_{j \neq i}\left(\lambda_{i}^{2}-\lambda_{j}^{2}\right)} \frac{1}{x+\lambda_{i}}\right)=
\end{gathered}
$$




$$
\sum_{i=1}^{p} \frac{\lambda_{i}^{2 p-2}}{2 \lambda_{i} \prod_{j \neq i}\left(\lambda_{i}^{2}-\lambda_{j}^{2}\right)}\left(\frac{1}{x-\lambda_{i}}-\frac{1}{x+\lambda_{i}}\right)=\sum_{i=1}^{p} \frac{\lambda_{i}^{2 p-2}}{\prod_{j \neq i}\left(\lambda_{i}^{2}-\lambda_{j}^{2}\right)}\left(\frac{1}{x^{2}-\lambda_{i}^{2}}\right) .
$$

This concludes the proof.

Lemma 6.3. If $\lambda_{1}, \lambda_{2}, \ldots, \lambda_{p}(p \geq 1)$ are distinct positive reals numbers, then

$$
\frac{x^{2 p-2}}{\prod_{i=1}^{p}\left(\lambda_{i}^{2}+x^{2}\right)}=\sum_{i=1}^{p} \frac{\lambda_{i}^{2 p-2}}{\prod_{j \neq i}\left(\lambda_{i}^{2}-\lambda_{j}^{2}\right)} \frac{1}{\lambda_{i}^{2}+x^{2}} .
$$

Proof: We will proceed by induction in $p$. For $p=1$, the equality is evident. Supose that the equality holds for $p$. Then, calculate

$$
\frac{x^{2 p}}{\prod_{i=1}^{p+1}\left(\lambda_{i}^{2}+x^{2}\right)}=\frac{x^{2 p-2}}{\prod_{i=1}^{p}\left(\lambda_{i}^{2}+x^{2}\right)} \frac{x^{2}}{\lambda_{p+1}^{2}+x^{2}}
$$

and by applying the hypothesis of induction, we deduce that (6.4) is equal to

$$
\begin{gathered}
\sum_{i=1}^{p} \frac{\lambda_{i}^{2 p-2}}{\prod_{j \neq i}\left(\lambda_{i}^{2}-\lambda_{j}^{2}\right)} \frac{1}{\lambda_{i}^{2}+x^{2}} \frac{x^{2}}{\lambda_{p+1}^{2}+x^{2}}= \\
\sum_{i=1}^{p} \frac{\lambda_{i}^{2 p-2}}{\left(\lambda_{i}^{2}-\lambda_{p+1}^{2}\right) \prod_{j \neq i}^{p}\left(\lambda_{i}^{2}-\lambda_{j}^{2}\right)}\left(\frac{\lambda_{i}^{2}}{\lambda_{i}^{2}+x^{2}}-\frac{\lambda_{p+1}^{2}}{\lambda_{p+1}^{2}+x^{2}}\right)= \\
\sum_{i=1}^{p} \frac{\lambda_{i}^{2 p-2}}{\left(\lambda_{i}^{2}-\lambda_{p+1}^{2}\right) \prod_{j \neq i}^{p}\left(\lambda_{i}^{2}-\lambda_{j}^{2}\right)}\left(\frac{\lambda_{i}^{2}}{\lambda_{i}^{2}+x^{2}}-\frac{\lambda_{p+1}^{2}}{\lambda_{p+1}^{2}+x^{2}}\right)= \\
\sum_{i=1}^{p} \frac{\lambda_{i}^{2 p}}{\prod_{j \neq i}^{p+1}\left(\lambda_{i}^{2}-\lambda_{j}^{2}\right)} \frac{1}{\lambda_{i}^{2}+x^{2}}-\sum_{i=1}^{p} \frac{\lambda_{p+1}^{2} \lambda_{i}^{2 p-2}}{\prod_{j \neq i}^{p+1}\left(\lambda_{i}^{2}-\lambda_{j}^{2}\right)} \frac{1}{\lambda_{p+1}^{2}+x^{2}} .
\end{gathered}
$$

Now, using Lemma 6.2 with $x=\lambda_{p+1}$, we obtain that

$$
\sum_{i=1}^{p} \frac{\lambda_{i}^{2 p-2}}{\prod_{j \neq i}^{p+1}\left(\lambda_{i}^{2}-\lambda_{j}^{2}\right)}=-\frac{\lambda_{p+1}^{2 p-2}}{\prod_{j=1}^{p}\left(\lambda_{p+1}^{2}-\lambda_{j}^{2}\right)}
$$

and then (6.5) is equal to

$$
\sum_{i=1}^{p+1} \frac{\lambda_{i}^{2 p}}{\prod_{j \neq i}^{p+1}\left(\lambda_{i}^{2}-\lambda_{j}^{2}\right)} \frac{1}{\lambda_{i}^{2}+x^{2}}
$$

This concludes the proof.

Proof of Theorem 2.14: First, we will prove the result for the case in which $\left\{X_{t}\right\}_{t \in \mathbb{R}} \sim \operatorname{FOU}\left(\lambda_{1}, \lambda_{2}, \ldots, \lambda_{p}, \sigma, H\right)$. Using property (5) of $f_{H}$ in (2.9) and Lemma 6.3 , we obtain that $\mathbb{E}\left(X_{0} X_{t}\right)=$ 


$$
\begin{gathered}
\frac{\sigma^{2} \Gamma(2 H+1) \sin (H \pi)}{2 \pi} \int_{-\infty}^{+\infty} e^{i t x}|x|^{1-2 H} \sum_{i=1}^{p} \frac{\lambda_{i}^{2 p-2}}{\prod_{j \neq i}\left(\lambda_{i}^{2}-\lambda_{j}^{2}\right)} \frac{1}{\lambda_{i}^{2}+x^{2}} d x \\
=\frac{\sigma^{2} \Gamma(2 H+1) \sin (H \pi)}{2 \pi} \int_{-\infty}^{+\infty} e^{i t x} \frac{|x|^{2 p-2 H-1}}{\prod_{i=1}^{p}\left(\lambda_{i}^{2}+x^{2}\right)} d x,
\end{gathered}
$$

and so (2.12) holds.

Now, in the general case in which $\left\{X_{t}\right\}_{t \in \mathbb{R}} \sim \mathrm{FOU}\left(\lambda_{1}^{\left(p_{1}\right)}, \lambda_{2}^{\left(p_{2}\right)}, \ldots, \lambda_{q}^{\left(p_{q}\right)}, \sigma, H\right)$ where $p_{1}+p_{2}+\ldots+p_{q}=p$, observe that for any $t, T_{\lambda+1 / n}\left(B_{H}\right)(t)=e^{-t / n} T_{\lambda}\left(B_{H}\right)(t)$ $\rightarrow T_{\lambda}\left(B_{H}\right)(t)$ when $n \rightarrow+\infty$. This fact allows us to obtain the auto-covariance function of $\operatorname{FOU}\left(\lambda^{(2)}, \sigma, H\right)$ as a limit of the auto-covariance function of any $\operatorname{FOU}(\lambda, \lambda+1 / n, \sigma, H)$. If we call $\rho_{\lambda, \lambda}$ and $\rho_{\lambda, \lambda+1 / n}$ the auto-covariance functions respectively, then using (2.12) and dominated convergence theorem, we obtain

$$
\rho_{\lambda, \lambda+1 / n}(t)=c_{H} \int_{-\infty}^{+\infty} \frac{e^{i t x}|x|^{3-2 H} d x}{\left(\lambda^{2}+x^{2}\right)\left((\lambda+1 / n)^{2}+x^{2}\right)} \rightarrow c_{H} \int_{-\infty}^{+\infty} \frac{e^{i t x}|x|^{3-2 H} d x}{\left(\lambda^{2}+x^{2}\right)^{2}} .
$$

Then, $\rho_{\lambda, \lambda}(t)=c_{H} \int_{-\infty}^{+\infty} \frac{e^{i t x}|x|^{3-2 H} d x}{\left(\lambda^{2}+x^{2}\right)^{2}}$ and $f(x)=c_{H} \frac{e^{i t x}|x|^{3-2 H}}{\left(\lambda^{2}+x^{2}\right)^{2}}$ is the espectral density of $\operatorname{FOU}\left(\lambda^{(2)}, \sigma, H\right)$. Using this argument repeatedly, we obtain that the autocovariance function of $\left\{X_{t}\right\}_{t \in \mathbb{R}}$ is the pointwise limit of the auto-covariance function of $\left\{X_{t}^{(n)}\right\}_{t \in \mathbb{R}} \sim \mathrm{FOU}(p)$ with parameters $\lambda_{1}, \lambda_{1}+1 / n, \ldots, \lambda_{1}+\left(p_{1}-1\right) / n, \ldots ., \lambda_{q}, \lambda_{q}+$ $1 / n, \ldots, \lambda_{q}+\left(p_{q}-1\right) / n, \sigma, H$. Then, from the fact that the spectral density of $\left\{X_{t}^{(n)}\right\}_{t \in \mathbb{R}}$ satisfies formula (2.12), we deduce that

$f^{\left(X^{(n)}\right)}(x) \rightarrow f^{(X)}(x)$ for all $x$, and so (2.11) holds. This concludes the proof.

Proof of Lemma 3.1:

$$
\begin{gathered}
\mathbb{E}\left(X_{t+s}^{(1)}-X_{s}^{(1)}\right)\left(X_{t+s}^{(2)}-X_{s}^{(2)}\right) \\
=\mathbb{E}\left(X_{t+s}^{(1)} X_{t+s}^{(2)}\right)+\mathbb{E}\left(X_{s}^{(1)} X_{s}^{(2)}\right)-\mathbb{E}\left(X_{t+s}^{(1)} X_{s}^{(2)}\right)-\mathbb{E}\left(X_{s}^{(1)} X_{t+s}^{(2)}\right) \\
=2 \mathbb{E}\left(X_{0}^{(1)} X_{0}^{(2)}\right)-\mathbb{E}\left(X_{t}^{(1)} X_{0}^{(2)}\right)-\mathbb{E}\left(X_{0}^{(1)} X_{t}^{(2)}\right) .
\end{gathered}
$$

We will work with the difference $\mathbb{E}\left(X_{0}^{(1)} X_{0}^{(2)}\right)-\mathbb{E}\left(X_{0}^{(1)} X_{t}^{(2)}\right)$, the other differences can be treated analogously.

Using (2.7) we can decompose

$$
\mathbb{E}\left(X_{0}^{(1)} X_{0}^{(2)}\right)-\mathbb{E}\left(X_{0}^{(1)} X_{t}^{(2)}\right)=A_{t}+B_{t}+C_{t}
$$

where

and

$$
\begin{gathered}
A_{t}:=\frac{\sigma^{2} H \Gamma(2 H)}{\lambda_{1}+\lambda_{2}}\left(\lambda_{2}^{1-2 H}\left(1-e^{-\lambda_{2} t}\right)+\lambda_{1}^{1-2 H}\left(1-e^{\lambda_{1} t}\right)\right), \\
B_{t}:=\frac{\sigma^{2} H}{\lambda_{1}+\lambda_{2}} \lambda_{1}^{1-2 H} e^{\lambda_{1} t} \int_{0}^{\lambda_{1} t} e^{-s} s^{2 H-1} d s
\end{gathered}
$$

$$
C_{t}:=\frac{\sigma^{2} H}{\lambda_{1}+\lambda_{2}} \lambda_{2}^{1-2 H} e^{-\lambda_{2} t} \int_{0}^{\lambda_{2} t} e^{s} s^{2 H-1} d s
$$


Using that $e^{-s}=1-e^{-c_{s}} s$ for $c_{t} \in\left(0, \lambda_{1} t\right)$ we obtain that

$$
B_{t}=\frac{\sigma^{2} H \lambda_{1}^{1-2 H} e^{\lambda_{1} t}}{\lambda_{1}+\lambda_{2}} \int_{0}^{\lambda_{1} t}\left(1-e^{-c_{s}} s\right) s^{2 H-1} d s=\frac{\sigma^{2} \lambda_{1}}{2\left(\lambda_{1}+\lambda_{2}\right)} t^{2 H}+o\left(t^{2 H}\right) .
$$

Analogoulsy, $C_{t}=\frac{\sigma^{2} \lambda_{2}}{2\left(\lambda_{1}+\lambda_{2}\right)} t^{2 H}+o\left(t^{2 H}\right)$. Then,

$$
B_{t}+C_{t}=\frac{\sigma^{2}}{2} t^{2 H}+o\left(t^{2 H}\right) .
$$

On the other hand, we can decompose

$$
\mathbb{E}\left(X_{0}^{(1)} X_{0}^{(2)}\right)-\mathbb{E}\left(X_{t}^{(1)} X_{0}^{(2)}\right)=A_{t}^{\prime}+B_{t}^{\prime}+C_{t}^{\prime}
$$

where

$$
\begin{gathered}
A_{t}^{\prime}:=\frac{\sigma^{2} H \Gamma(2 H)}{\lambda_{1}+\lambda_{2}}\left(\lambda_{2}^{1-2 H}\left(1-e^{\lambda_{2} t}\right)+\lambda_{1}^{1-2 H}\left(1-e^{-\lambda_{1} t}\right)\right), \\
B_{t}^{\prime}:=\frac{\sigma^{2} H}{\lambda_{1}+\lambda_{2}} \lambda_{2}^{1-2 H} e^{\lambda_{2} t} \int_{0}^{\lambda_{2} t} e^{-s} s^{2 H-1} d s
\end{gathered}
$$

and

$$
C_{t}^{\prime}:=\frac{\sigma^{2} H}{\lambda_{1}+\lambda_{2}} \lambda_{1}^{1-2 H} e^{-\lambda_{1} t} \int_{0}^{\lambda_{1} t} e^{s} s^{2 H-1} d s .
$$

Then, $B_{t}^{\prime}+C_{t}^{\prime}=\frac{\sigma^{2}}{2} t^{2 H}+o\left(t^{2 H}\right)$.

Thus,

$$
\begin{gathered}
2 \mathbb{E}\left(X_{0}^{(1)} X_{0}^{(2)}\right)-\mathbb{E}\left(X_{t}^{(1)} X_{0}^{(2)}\right)-\mathbb{E}\left(X_{0}^{(1)} X_{t}^{(2)}\right)=A_{t}+B_{t}+C_{t}+A_{t}^{\prime}+B_{t}^{\prime}+C_{t}^{\prime}= \\
A_{t}+A_{t}^{\prime}+\sigma^{2} t^{2 H}+o\left(t^{2 H}\right) .
\end{gathered}
$$

To finish the proof, it is enough to see that $A_{t}+A_{t}^{\prime}=o\left(t^{2 H}\right)$. Indeed,

$$
\begin{aligned}
& A_{t}+A_{t}^{\prime} \\
& =\frac{\sigma^{2} H \Gamma(2 H)}{\lambda_{1}+\lambda_{2}}\left(\lambda_{2}^{1-2 H}\left(2-e^{-\lambda_{2} t}-e^{\lambda_{2} t}\right)+\lambda_{1}^{1-2 H}\left(2-e^{-\lambda_{1} t}-e^{\lambda_{1} t}\right)\right)=o\left(t^{2 H}\right) .
\end{aligned}
$$

This concludes the proof.

Proof of Theorem 3.2: Observe that it is enough to prove the result for the case in which all the $\lambda_{i}$ are distinct. This is because in the general case, any $\operatorname{FOU}(p)$ is pointwise limit of a sequence of $\mathrm{FOU}(p)$ where the parameters are pairwise distinct, and then we use a similar argument to the one used in the proof of Theorem 2.14.

Now, suppose that $X_{t}=\sum_{i=1}^{p} K_{i}(\lambda) X_{t}^{(i)}$ where $K_{i}(\lambda)$ are defined in (2.2), and define $v_{i}(t):=\frac{1}{2} \mathbb{E}\left(X_{t+s}^{(i)}-X_{s}^{(i)}\right)^{2}$ the variogram of each process $\left\{X_{t}^{(i)}\right\}_{t \in \mathbb{R}}$. To simplify the notation, we write $K_{i}$ instead of $K_{i}(\lambda)$.

We start with

$$
\begin{gathered}
v(t)=\frac{1}{2} \mathbb{E}\left(X_{t+s}-X_{s}\right)^{2}=\frac{1}{2} \mathbb{E}\left[\sum_{i=1}^{p} K_{i}\left(X_{t+s}^{(i)}-X_{s}^{(i)}\right)\right]^{2}= \\
\frac{1}{2} \sum_{i=1}^{p} K_{i} \mathbb{E}\left(X_{t+s}^{(i)}-X_{s}^{(i)}\right)^{2}+\frac{1}{2} \mathbb{E} \sum_{i \neq j=1}^{p} K_{i} K_{j}\left(X_{t+s}^{(i)}-X_{s}^{(i)}\right)\left(X_{t+s}^{(j)}-X_{s}^{(j)}\right)
\end{gathered}
$$


Now, using that $v_{i}(t)=\frac{\sigma^{2}}{2}|t|^{2 H}+o\left(|t|^{2 H}\right)$ and Lemma 3.1, we obtain that (6.6) is equal to

$$
\sum_{i=1}^{p} K_{i}^{2}\left[\frac{\sigma^{2}}{2}|t|^{2 H}+o\left(|t|^{2 H}\right)\right]+\frac{1}{2} \mathbb{E} \sum_{i \neq j=1}^{p} K_{i} K_{j}\left[\sigma^{2}|t|^{2 H}+o\left(|t|^{2 H}\right)\right]
$$

and using that $\sum_{i=1}^{p} K_{i}=1$ we obtain that (6.7) is equal to

$$
\frac{\sigma^{2}}{2}|t|^{2 H}+o\left(|t|^{2 H}\right) \text {. }
$$

This concludes the proof.

Proof of Theorem 3.5: We verify the hypotheses of Theorem 3 (i) and (iii) in Istas and Lang (1997). We do not follow strictly the notation of Istas \& Lang. The writing of this theorem is adapted to the notation of our work. The theorem start with the assumptions $(A 1)$ and $(A 2)$ that we show below.

Assumption $(A 1)$

Denote $D$ the greatest integer such that $v$ is $2 D$ times differentiable. We assume that there exists a real $s$ such that $0<s<2$ and a real $C>0$ such that:

$$
v^{(2 D)}(t)=v^{(2 D)}(0)+C(-1)^{D}|t|^{s}+r(t) \text { and } r(t)=o\left(|t|^{s}\right) \text { at zero. }
$$

Assumption $(A 2)$

Given a filter $a=\left(a_{0}, a_{1}, \ldots, a_{p}\right)$, we assume that for any real $s$ such that $0<$ $s<2 M(a)$ and $s$ is not an even integer:

$$
\sum_{k=0}^{p} \sum_{l=0}^{p} a_{k} a_{l}|k-l|^{s} \neq 0
$$

where $M(a)$ denotes the order of the first non-zero moment of the filter $a$. It is defined by:

$$
\sum_{i=0}^{p} a_{i} i^{k}=0 \text { for } 0 \leq k<M(a) \text { and } \sum_{i=0}^{p} a_{i} i^{M(a)} \neq 0 .
$$

\section{Theorem 3 (i) (Istas \& Lang)}

- Let $X$ be a centred process with stationary increments satisfying the condition $(A 1)$.

- Let $\alpha>0$ and define the observation mesh $\Delta$ by $\Delta(n)=n^{-\alpha}$.

- Consider several filters $a^{1}, a^{2}, \ldots, a^{I}$. We denote $p^{i}$ the length of the sequence $a^{i}$ and define the matrix $A$ with size $I \times p$ where $p=\max \left\{p^{1}, p^{2}, \ldots, p^{I}\right\}$ by $A_{i j}=2 \sum_{k=0}^{p^{i}-j} a_{k}^{i} a_{k+j}^{i}$ for $j=1, \ldots, p^{i}$ and $A_{i j}=0$ otherwise. Assume that the $a^{i}$ are such $A$ is full rank.

- Assume that there exist three reals $\delta, G>0, \gamma>s$ and an integer $q>$ $\gamma+1 / 2$, such that the remainder $r(t)$ is $q$ times differentiable on $(0, \delta]$ and $\left|r^{(q)}(t)\right| \leq G|t|^{\gamma-q}$. If $\delta<T$, assume that for some integer $d>s+1 / 2, v$ is $2 D+d$ times differentiable on $(\delta, T]$ and that

$$
\int_{\delta}^{T}\left|v^{(2 D+d)}(t)\right| d t<+\infty
$$

We choose a filter $a$ satisfying $(A 2)$ with $2 M(a) \geq \max \{2 D+q, 2 D+d\}$. 
Then, as $n \rightarrow+\infty$,

$$
(\widehat{h}, \widehat{C}) \stackrel{a . s .}{\rightarrow}(h, C) .
$$

\section{Theorem 3 (iii) (Istas \& Lang)}

- Let $X$ be a centred process with stationary increments satisfying the condition $(A 1)$.

- Let $\alpha>0$ and define the observation mesh $\Delta$ by $\Delta(n)=n^{-\alpha}$.

- Consider several sequences $a^{1}, a^{2}, \ldots, a^{I}$. We denote $p^{i}$ the length of the sequence $a^{i}$ and define the matrix $A$ with size $I \times p$ where $p=\max \left\{p^{1}, p^{2}, \ldots\right.$, $\left.p^{I}\right\}$ by $A_{i j}=2 \sum_{k=0}^{p^{i}-j} a_{k}^{i} a_{k+j}^{i}$ for $j=1, \ldots, p^{i}$ and $A_{i j}=0$ otherwise. Assume that the $a^{i}$ are such $A$ is full rank.

- Assume that there exist three reals $\delta, G>0, \gamma>s$ and an integer $q \geq 2$, greater than $\gamma+1 / 2$, such that the remainder $r(t)$ is $q$ times differentiable on $(0, \delta]$ and $\left|r^{(q)}(t)\right| \leq G|t|^{\gamma-q}$. If $\delta<T$, assume that for some integer $d \geq 2$, greater than $s+1 / 2, v$ is $2 D+d$ times differentiable on $(\delta, T]$ and that

$$
\int_{\delta}^{T}\left|v^{(2 D+d)}(t)\right| d t<+\infty .
$$

We choose a filter $a$ satisfying $(A 2)$ with $2 M(a) \geq \max \{2 D+q, 2 D+d\}$. - If $s>1$, we choose $\Delta(n)$ such that

$$
n \Delta^{2(s-1)}(n) \rightarrow+\infty \text {. }
$$

Then, as $n \rightarrow+\infty$,

$$
\begin{aligned}
& \sqrt{n}(\widehat{h}-h) \text { converges in distribution to a centred Gaussian variable. } \\
& \frac{\sqrt{n}}{\log n}(\widehat{C}-C) \text { converges in distribution to a centred Gaussian variable. }
\end{aligned}
$$

From Theorem 3.2, we obtain that $v(t)=\frac{\sigma^{2}}{2}|t|^{2 H}+r(t)$ where $r(t)=o\left(|t|^{2 H}\right)$ when $t \rightarrow 0$. Then $h=2 H$ and $C=\sigma^{2} / 2$. We use $I=2$ in condition $(A 2)$ by taking a filter $a$ and $a^{2}$ defined in Definition 25. Further, in the proof of property 4 in Proposition 2.7, we see that there exists $G>0$ such that

$$
\left|r^{(4)}(t)\right| \leq G|t|^{2 H+1-\varepsilon-4} \text { for } t \in(0,1)
$$

holds for any $H, \varepsilon \in(0,1)$. In the other hand, observe that in our case $s=2 H>1$ (because $H>1 / 2$ ) and condition (6.8) is fulfilled by taking $\Delta_{n}=n^{-\alpha}$ for $\alpha$ such that $0<\alpha<\frac{1}{2(2 H-1)}$. This concludes the proof.

Proof of Theorem 3.6: The formula for the spectral density (2.11) and the choice of the weight function $w$, allow us to verify the conditions A.I to A.V of Leonenko and Sakhno (2006) which demonstrates the consistency and asymptotic normality of our estimators. Conditions A.I to A.V are the following:

A. I. Let $Y(t), t \in[0, T]$, be an observation of a real-value measurable stationary Gaussian process $Y(t), t \in \mathbb{R}$ with zero mean and spectral density $f(x, \lambda) x \in \mathbb{R}$, $\lambda \in \Lambda \subset \mathbb{R}^{m}$, where $\Lambda$ is a compact set, and the true value of the parameter 
$\lambda^{0} \in \operatorname{int}(\Lambda)$, the interior of $\Lambda$. Suppose further than $f(x, \lambda) \neq f\left(x, \lambda^{\prime}\right)$ for $\lambda \neq \lambda^{\prime}$ almost everywhere in $\mathbb{R}$ with respect to the Lebesgue measure.

Consider $w(x)$ a symmetric about $x=0$ function such that $U_{T}(\lambda)$ defined in (3.6) is well defined.

A.II. $f\left(x, \lambda^{0}\right) w(x) / f(x, \lambda) \in L^{1}(\mathbb{R}) \cap L^{2}(\mathbb{R})$ for all $\lambda \in \Lambda$.

A.III. There exists a function $v(x), x \in \mathbb{R}$ such that:

(i) The function $h(x, \lambda)=v(x) / f(x, \lambda)$ is uniformly continuous in $\mathbb{R} \times \Lambda$;

(ii) $f\left(x, \lambda^{0}\right) w(x) / v(x) \in L^{1}(\mathbb{R}) \cap L^{2}(\mathbb{R})$.

A.IV. The function $1 / f(x, \lambda)$ is twice differentiable in a neigborhood of the point $\lambda^{0}$ and

(i) $f\left(x, \lambda^{0}\right) w(x)\left(\partial^{2} / \partial \lambda_{i} \partial \lambda_{j}\right)(1 / f(x, \lambda)) \in L^{1}(\mathbb{R}) \cap L^{2}(\mathbb{R}), i, j=1,2, \ldots, m$, $\lambda \in \Lambda$;

(ii) $f\left(x, \lambda^{0}\right) w(x)\left(\partial / \partial \lambda_{i}\right)(1 / f(x, \lambda)) \in L^{k}(\mathbb{R})$ for all $k \geq 1, i=1,2, \ldots, m, \lambda \in \Lambda$;

(iii) $\sqrt{T} \int_{\mathbb{R}} \mathbb{E}\left(I_{T}(x)-f\left(x, \lambda^{0}\right)\right) w(x)\left(\partial / \partial \lambda_{i}\right)(1 / f(x, \lambda)) d x \rightarrow 0$ as $T \rightarrow+\infty$ for all $i=1,2, \ldots, m, \lambda \in \Lambda$.

A.V. The matrices $W_{1}(\lambda)$ and $W_{2}(\lambda)$ are positive definite.

Theorem 1 and Theorem 2 in Leonenko and Sakhno (2006), prove that under the conditions A.I to A.V it holds that

- $\hat{\lambda}_{T} \stackrel{P}{\rightarrow} \lambda^{0}$ when $T \rightarrow+\infty$ and

- $\sqrt{T}\left(\hat{\lambda}_{T}-\lambda^{0}\right) \stackrel{w}{\rightarrow} N_{q}\left(0, W_{1}^{-1}\left(\lambda^{0}\right) W_{2}\left(\lambda^{0}\right) W_{1}^{-1}\left(\lambda^{0}\right)\right)$ when $T \rightarrow+\infty$.

In our case $m=q$ and we will check conditions A.I to A.V for our process.

Conditions A.I, A.II and A.IV are verified directly. For condition A.III, it is enough to take $\alpha$ such that $\|\lambda\|^{2} \leq \alpha$ for all $\lambda \in \Lambda$ and consider the function $v(x)=\frac{|x|^{(2 p-1-2 H)}}{\left(\alpha+x^{2}\right)^{p}}$. To conclude the proof we will show now that the matrices $W_{1}(\lambda)$ and $W_{2}(\lambda)$ are positive definite (condition A.V).

We work in $L^{2}(w(x) d x)$, so that $\langle f, g\rangle=\int_{-\infty}^{+\infty} w(x) f(x) g(x) d x$. Fixed $\lambda$, for each $z \in \mathbb{R}^{q}$ we obtain that

$$
\begin{gathered}
\left\|\sum_{i=1}^{q} \frac{\partial}{\partial \lambda_{i}} \log f^{(X)}(., \lambda) z_{i}\right\|^{2}= \\
\sum_{i, j=1}^{q}\left\langle\frac{\partial}{\partial \lambda_{i}} \log f^{(X)}(., \lambda), \frac{\partial}{\partial \lambda_{j}} \log f^{(X)}(., \lambda)\right\rangle z_{i} z_{j}=\sum_{i, j=1}^{q} w_{i j}^{(1)}(\lambda) z_{i} z_{j} .
\end{gathered}
$$

Then $\sum_{i, j=1}^{q} w_{i j}^{(1)}(\lambda) z_{i} z_{j} \geq 0$ for all $z \in \mathbb{R}^{q}$. Up to this point the formula for $f^{(X)}(x, \lambda)$ has not been necessary.

If $\sum_{i, j=1}^{q} w_{i j}^{(1)}(\lambda) z_{i} z_{j}=0$ then $\sum_{i=1}^{q} \frac{\partial}{\partial \lambda_{i}} \log f^{(X)}(., \lambda) z_{i}=0$ and it follows that (using that $w>0$ and the formula for the spectral density) $\sum_{i=1}^{q} \frac{p_{i} \lambda_{i} z_{i}}{\lambda_{i}^{2}+x^{2}}=0$ for all $x \neq 0$. Writing $a_{i}=p_{i} \lambda_{i} z_{i}$ for each $i=1,2,3, \ldots, q$, and taking the derivative $q$ times in the equality $\sum_{i=1}^{q} \frac{a_{i}}{\lambda_{i}^{2}+x^{2}}=0$, and taking limit when $x \rightarrow 0$, we obtain $\left\{\begin{array}{c}\sum_{i=1}^{q} \alpha_{i} a_{i}=0 \\ \sum_{i=1}^{q} \alpha_{i}^{2} a_{i}=0 \\ \vdots \\ \sum_{i=1}^{q} \alpha_{i}^{q} a_{i}=0\end{array}\right.$

where $\alpha_{i}=\frac{1}{\lambda_{i}^{2}}$. As the $\lambda_{i}^{\prime} s$ are distinct we may use well known facts about the Vandermonde determinant, to deduce that $a_{1}=a_{2}=\ldots=a_{q}=0$, thus $z_{1}=z_{2}=$ 
$\ldots=z_{q}=0$ and the matrix $W_{1}(\lambda)$ is positive definite for each $\lambda$. Analogously, working in $L^{2}\left(w^{2}(x) d x\right)$ yields that $W_{2}(\lambda)$ is positive definite. This concludes the proof.

\section{Acknowledgements}

The authors would like to thank an anonymous referee for the carefully lecture of our paper, for his proposals and for suggesting several changes that substantially improved our work. We also thank Enrique Cabaña and Jorge Graneri for their generosity and for contributing ideas for this work.

\section{References}

B. Ai, Y. He and W. Zhong. Transport in periodic potentials induced by fractional Gaussian noise. Phys. Rev. E 82, 061102 (2010). DOI: 10.1103/PhysRevE.82.061102.

A. Arratia, A. Cabaña and E. M. Cabaña. A construction of continuous-time ARMA models by iterations of Ornstein-Uhlenbeck processes. SORT 40 (2), 267-302 (2016). MR3592490.

P. Cheridito, H. Kawaguchi and M. Maejima. Fractional Ornstein-Uhlenbeck processes. Electron. J. Probab. 8, no. 3, 14 (2003). MR1961165.

D. Delignières, K. Torre and P. L. Bernard. Transition from Persistent to Anti-Persistent Correlations in Postural Sway Indicates Velocity-Based Control. PLoS Computational Biology. 7 (2), 1001089 (2011). DOI: 10.1371/journal.pcbi.1001089.

I. A. Ibragimov and Y. A. Rozanov. Gaussian random processes, volume 9 of Applications of Mathematics. Springer-Verlag, New York-Berlin (1978). ISBN 0-387-90302-X. MR543837.

J. Istas and G. Lang. Quadratic variations and estimation of the local Hölder index of a Gaussian process. Ann. Inst. H. Poincaré Probab. Statist. 33 (4), 407-436 (1997). MR1465796.

T. Kaarakka and P. Salminen. On fractional Ornstein-Uhlenbeck processes. Commun. Stoch. Anal. 5 (1), 121-133 (2011). MR2808539.

P. Langevin. Sur la théorie du mouvement brownien. C.R. Acad. Sci. París 146, 530-533 (1908).

N. N. Leonenko and L. M. Sakhno. On the Whittle estimators for some classes of continuous-parameter random processes and fields. Statist. Probab. Lett. 76 (8), 781-795 (2006). MR2266092.

V. Maxim, L. Sendur, J. L. Fadili, J. Sucking, R. Gould, Howard. R. and E. Bullmore. Fractional Gaussian noise, functional MRI and Alzheimer's disease. NeuroImage, Elsevier 25 (1), 141-158 (2005). DOI: 10.1016/j.neuroimage.2004.10.044.

V. Pipiras and M. S. Taqqu. Integration questions related to fractional Brownian motion. Probab. Theory Related Fields 118 (2), 251-291 (2000). MR1790083.

G. E. Uhlenbeck and L. S. Ornstein. On the theory of Brownian Motion. Physical Review 236, 823-841 (1930). 